Pacific Journal of Mathematics

SOME INFINITE CHAINS IN THE LATTICE OF VARIETIES OF 


\title{
SOME INFINITE CHAINS \\ IN THE LATTICE OF VARIETIES OF INVERSE SEMIGROUPS
}

\section{David CowaN}

\begin{abstract}
The relation $\nu$ defined on the lattice $\mathscr{L}(\mathscr{J})$ of varieties of inverse semigroups by $\mathscr{U} \nu \mathscr{V}$ if and only if $\mathscr{U} \cap \mathscr{G}=\mathscr{V} \cap \mathscr{G}$ and $\mathscr{U} \vee \mathscr{G}=\mathscr{V} \vee \mathscr{G}$, where $\mathscr{G}$ is the variety of groups, is a congruence. It is known that varieties belonging to the first three layers of $\mathscr{L}(\mathscr{I})$ (those varieties belonging to the lattice $\mathscr{L}(\mathscr{S} \mathscr{F})$ of varieties of strict inverse semigroups) possess trivial $\nu$-classes and that there exist non-trivial $\nu$-classes in the next layer of $\mathscr{L}(\mathscr{J})$. We show that there are infinitely many $\nu$-classes in the fourth layer of $\mathscr{L}(\mathscr{I})$, and also higher up in $\mathscr{L}(\mathscr{I})$, that in fact contain an infinite descending chain of varieties. To find these chains we first construct a collection of semigroups in $\mathscr{B}^{1}$, the variety generated by the five element combinatorial Brandt semigroup with an identity adjoined. By considering wreath products of abelian groups and these semigroups from $\mathscr{B}^{1}$, we obtain an infinite descending chain in the $\nu$-class of $\mathscr{U} \vee \mathscr{B}^{1}$, for every non-trivial abelian group variety $\mathscr{U}$.
\end{abstract}

1. Introduction. In [K1] Kleiman demonstrated that the relation $\nu$ defined on the lattice $\mathscr{L}(\mathscr{I})$ of varieties of inverse semigroups by $\mathscr{U} \nu \mathscr{V}$ if and only if $\mathscr{U} \cap \mathscr{G}=\mathscr{V} \cap \mathscr{G}$ and $\mathscr{U} \vee \mathscr{G}=\mathscr{V} \vee \mathscr{G}$, where $\mathscr{G}$ is the variety of groups, is a congruence. He further showed that the lattice $\mathscr{L}(\mathscr{S} \mathscr{J})$ of varieties of strict inverse semigroups is isomorphic to three copies of the lattice $\mathscr{L}(\mathscr{G})$ of varieties of groups and that each of the intervals $[\mathscr{S}, \mathscr{S} \vee \mathscr{G}]$ and $[\mathscr{B}, \mathscr{B} \vee \mathscr{G}$, where $\mathscr{S}$ is the variety of semilattices and $\mathscr{B}$ is the variety generated by the five element combinatorial Brandt semigroup, is isomorphic to $\mathscr{L}(\mathscr{G})$ (and so, as a result, $\mathscr{L}(\mathscr{S} \mathscr{S})$ is a modular lattice). Consequently, for any variety $\mathscr{V}$ in $\mathscr{L}(\mathscr{S} \mathscr{F})$, the $\nu$-class of $\mathscr{V}$ is trivial. $\mathscr{L}(\mathscr{S} \mathscr{F})$ is sometimes referred to colloquially as the first three layers of the lattice $\mathscr{L}(\mathscr{I})$. The "fourth" layer, $\left[\mathscr{B}^{1}, \mathscr{B}^{1} \vee \mathscr{G}\right]$, where $\mathscr{B}^{1}$ is the variety generated by the five element combinatorial Brandt semigroup with an identity adjointed, is not nearly as nice. While it is a modular lattice (the collection of congruences on an inverse semigroup which have the same trace forms a complete modular sublattice of the lattice of congruences on that semigroup), the $\nu$-classes of its members are not all 
trivial and, as a result, $\mathscr{L}\left(\mathscr{B}^{1} \vee \mathscr{G}\right)$ is not modular, and hence $\mathscr{L}(\mathscr{I})$ is not modular (Reilly [R2] provides an example which demonstrates this). In this note we show that the $\nu$-class of $\mathscr{B}^{1} \vee \mathscr{A}$, for any abelian group variety $\mathscr{A}$, contains an infinite chain of varieties and so is far from being trivial. The technique used is interesting in that we are only required to know the structure of the $\mathscr{D}$-classes (as reflected by their Schützenberger graphs) of a given collection of words with respect to $\mathscr{B}^{1}$ (and not the entire $\mathscr{B}^{1}$-free object on countably infinite $X$ ) in order to construct inverse semigroups which are then shown to generate distinct varieties. We remark that the variety $\mathscr{B}^{1}$ has proved to be rather enigmatic. Even though it is generated by a small (6-element) inverse semigroup and $\mathscr{L}\left(\mathscr{B}^{1}\right)$ is just a 4-element chain, its members are not easily characterized and, as Kleiman proved in [K2], it is not defined by a finite set of identities.

Section 2 is devoted to preliminary material. In $\S 3$ we construct a collection of inverse semigroups each of which belongs to the variety $\mathscr{B}^{1}$ but not $\mathscr{B}$. From these semigroups we construct in $\S 4$ a collection of inverse semigroups belonging to $\mathscr{B}^{1} \circ \mathscr{A}_{n}, n \in \omega$, but not $\mathscr{B}^{1} \vee \mathscr{A}_{n}$. In the final section we use the semigroups of $\S 4$ to construct an infinite chain of varieties in the interval $\left[\mathscr{B}^{1} \vee \mathscr{A}_{n}, \mathscr{A}_{n} \circ \mathscr{B}^{1}\right]$ which is the $\nu$ class of $\mathscr{B}^{1} \vee \mathscr{A}_{n}$ (by a theorem due to Reilly [R1]). Using this result we can then show that a larger collection of $\nu$-classes which are also intervals in $\mathscr{L}(\mathscr{I})$ possess an infinite descending chain of varieties.

2. Preliminaries. We assume that the reader is familiar with the basic notions of inverse semigroup theory for which Petrich $[\mathbf{P}]$ is a standard reference. For the basic results concerning varieties we refer the reader to [BS]. We will consistently use the following notation:

$\mathscr{I}-$ the variety of all inverse semigroups

$\mathscr{G}$ - the variety of groups

$B_{2}$ - the five element combinatorial Brandt semigroup

$\mathscr{B}$ - the variety generated by the five element combinatorial Brandt semigroup $B_{2}$; it is defined by the identity $x y x^{-1}=$ $\left(x y x^{-1}\right)^{2}$

$B_{2}^{1}-B_{2}$ with an identity adjoined

$\mathscr{B}^{1}$ - the variety generated by $B_{2}^{1}$

$\mathscr{A}$ - the variety of abelian groups

$\mathscr{A}_{n}$ - the variety of abelian groups of exponent $n$ 
$F \mathscr{U}(X)$ - the $\mathscr{U}$-free object on $X$ in the variety $\mathscr{U}$

$\rho(\mathscr{U})$ - the fully invariant congruence on $F \mathscr{F}(X)$ corresponding to the variety $\mathscr{U}$

$c(w)$ - for any $w$ over $X \cup X^{-1}$, the content of $w$ which is the set $\left\{x \in X: x\right.$ or $x^{-1}$ occurs in $\left.w\right\}$

$w \in E-$ for a word $w$ over $X \cup X^{-1}$, the identity $w=w^{2}$

Throughout this note $X=\left\{x_{i}: i \in \omega\right\}$ is a fixed countably infinite set.

For any congruence $\rho$ on an inverse semigroup $S$, define the kernel of $\rho, \operatorname{ker} \rho$, and the trace of $\rho, \operatorname{tr} \rho$, by

$$
\begin{aligned}
\operatorname{ker} \rho & =\{s \in S: s \rho e \text { for some idempotent } e \text { in } S\} \\
& =\left\{s \in S: s \rho s^{2}\right\}=\left\{s \in S: s \rho=(s \rho)^{2}\right\}, \\
\operatorname{tr} \rho & =\rho \cap\left(E_{S} \times E_{S}\right) .
\end{aligned}
$$

Every congruence $\rho$ on an inverse semigroup $S$ is completely determined by its kernel and trace, [P; III.1.5].

An inverse semigroup $S$ is combinatorial if $\mathscr{H}=\varepsilon$ in $S$. The variety $\mathscr{V}$ is said to be combinatorial if all members of $\mathscr{V}$ are combinatorial. The variety $\mathscr{B}^{1}$ is a combinatorial variety. Moreover, $\mathscr{B}^{1} \subseteq \mathscr{U}^{\max }=\left[w=w^{2}: w=w^{2}\right.$ is a law in $\left.\mathscr{U}\right]$ for all group varieties $\mathscr{U}$ (see [PR]).

Let $S$ be an inverse semigroup. A transformation $\rho$ on $S$ is a right translation of $S$ if, for all $x, y \in S,(x y) \rho=x(y \rho)$. Likewise, a transformation $\lambda$ is a left translation if $\lambda(x y)=(\lambda x) y$, for all $x, y \in$ $S$. If, in addition, the left translation $\lambda$ and the right translation $\rho$ satisfy $x(\lambda y)=(x \rho) y$, for all $x, y \in S$, then the two are linked and the pair $(\lambda, \rho)$ is a bitranslation. The set of all bitranslations on $S$ under the operation of componentwise composition is an inverse semigroup and is called the translational hull of $S$ [P; V.1.4]. We denote this semigroup by $\Omega(S)$.

For any $s \in S$, the functions $\lambda_{s}$ and $\rho_{s}$ defined by $\lambda_{s} x=s x$ and $x \rho_{s}=x s$, for all $x \in S$, are left and right translations, respectively. In fact, $\left(\lambda_{s}, \rho_{s}\right)$ is a bitranslation and so is a member of $\Omega(S)$. The mapping

$$
\pi: s \rightarrow\left(\lambda_{s}, \rho_{s}\right) \quad(s \in S)
$$

is a monomorphism of $S$ into $\Omega(S)$ and is called the canonical homomorphism of $S$ into $\Omega(S)$. 
If $S$ is an ideal of the inverse semigroup $V$ then $V$ is an ideal extension of $S$ (by the Rees quotient semigroup $V / S$ ).

Let $V$ be an ideal extension of $S$. For each $v \in V$, define

$$
\lambda^{v} s=v s \quad \text { and } \quad s \rho^{v}=s v \quad(s \in S) .
$$

Then the mapping

$$
\tau(V: S): V \rightarrow \Omega(S)
$$

defined by

$$
v \tau(V: S)=\left(\lambda^{v}, \rho^{v}\right) \quad(v \in V)
$$

is a homomorphism of $V$ into $\Omega(S)$ which extends $\pi$. Moreover, $\tau(V: S)$ is the unique extension of $\pi$ to a homomorphism of $V$ into $\Omega(S)$ [P; I.9.2]. We call $\tau(V: S)$ the canonical homomorphism of $V$ into $\Omega(S)$.

Let $S$ and $T$ be inverse semigroups and suppose that $T$ is an inverse subsemigroup of $\mathscr{I}(I)$, the symmetric inverse semigroup on $I$. Let ${ }^{I} S$ denote the set of functions (written on the right) from subsets of $I$ into $S$. For any $\psi \in{ }^{I} S$, denote the domain of $\psi$ by d $\psi$. Define a multiplication on ${ }^{I} S$ by

$$
i\left(\psi \cdot \psi^{\prime}\right)=(i \psi) \cdot\left(i \psi^{\prime}\right) \quad\left[i \in \mathbf{d} \psi \cap \mathbf{d} \psi^{\prime}\right] .
$$

For any $\beta \in \mathscr{I}(I)$ and $\psi \in{ }^{I} S$, we define a mapping ${ }^{\beta} \psi$ by

$$
i\left({ }^{\beta} \psi\right)=(i \beta) \psi \quad[i \in \mathbf{d} \beta, i \beta \in \mathbf{d} \psi] .
$$

The (right) wreath product of $S$ and $T$ is the set

$$
S \mathrm{wr} T=\left\{(\psi, \beta) \in{ }^{I} S \times T: \mathbf{d} \psi=\mathbf{d} \beta\right\}
$$

with multiplication given by

$$
(\psi, \beta) \cdot\left(\psi^{\prime}, \beta^{\prime}\right)=\left(\psi^{\beta} \psi^{\prime}, \beta \beta^{\prime}\right) .
$$

If $T$ is an inverse subsemigroup of $\mathscr{I}(I)$, we will sometimes write $(T, I)$ for $T$ if we wish to emphasize the set $I$ on which $T$ acts.

Our definition of wreath product follows that of Houghton [H]. In [H] the wreath product $W(S, T)$ of inverse semigroups $S$ and $T$ is, in our notation, $S \mathrm{wr}(T, T)$ where $T$ is given the Wagner representation by partial right translations. Our notation follows Petrich [P; V.4]. It is not difficult to verify that if $S$ and $(T, I)$ are inverse semigroups then $S \mathrm{wr}(T, I)$ is also an inverse semigroup. In fact, if $(\psi, \beta) \in S \mathrm{wr}(T, I)$ then

$$
(\psi, \beta)^{-1}=\left(\psi^{-1}, \beta^{-1}\right)
$$


where $\psi^{-1} \in{ }^{I} S$ and $\beta^{-1} \in T$ are defined by

$$
\begin{gathered}
\mathbf{d} \beta^{-1}=\mathbf{d} \psi^{-1}=\{i \beta: i \in \mathbf{d} \beta\}, \\
\beta^{-1} \text { is the inverse of } \beta \text { in } T \text { and } \\
i \psi^{-1}=\left(i \beta^{-1} \psi\right)^{-1} \quad\left(i \in \mathbf{d} \beta^{-1}\right) .
\end{gathered}
$$

Equivalently, we may define $\psi^{-1}$ by

$$
j \beta \psi^{-1}=(j \psi)^{-1} \quad(j \in \mathbf{d} \beta) .
$$

For any $(\psi, \beta)$ belonging to $S \mathrm{wr}(T, I)$, we have written $(\psi, \beta)^{-1}$ as $\left(\psi^{-1}, \beta^{-1}\right)$ even though the definition of $\psi^{-1}$ depends upon $\beta$. This is not to suggest that if $\left(\psi, \beta^{\prime}\right)$ is another member of $S \mathrm{wr}(T, I)$, then the first coordinate of $\left(\psi, \beta^{\prime}\right)^{-1}$ is the same as the first coordinate of $(\psi, \beta)^{-1}$. We use $\psi^{-1}$ to avoid notational difficulties and simply note that when $\psi^{-1}$ is used, the member of $(T, I)$ to which it is paired will be understood.

Let $\mathscr{U}$ and $\mathscr{V}$ be varieties of inverse semigroups. The Mal'cev product of $\mathscr{U}$ and $\mathscr{V}$, denoted by $\mathscr{U} \circ \mathscr{V}$, is the collection of those inverse semigroups $S$ for which there exists a congruence $\rho$ on $S$ with the property that $e \rho \in \mathscr{U}$ for all $e \in E_{S}$ and $S / \rho \in \mathscr{V}$. In general, $\mathscr{U} \circ \mathscr{V}$ is not a variety. For example, if $\mathscr{V}$ is any nontrivial group variety and $\mathscr{U}=\mathscr{S}$ then the five element combinatorial Brandt semigroup $B_{2}$ is a member of $\langle\mathscr{U} \circ \mathscr{V}\rangle$ but $B_{2}$ is not a member of $\mathscr{U} \circ \mathscr{V}$. However, when $\mathscr{U}$ is a variety of groups, $\mathscr{U} \circ \mathscr{V}$ is a variety (see [P; XII 8.3] or [B]). Note that, if $\mathscr{V}$ and $\mathscr{W}$ are varieties such that $\mathscr{V} \subseteq \mathscr{W}$ then, for any variety $\mathscr{U}, \mathscr{U} \circ \mathscr{V} \subseteq \mathscr{U} \circ \mathscr{W}$ and $\mathscr{V} \circ \mathscr{U} \subseteq$ $\mathscr{W} \circ \mathscr{U}$.

$\mathrm{Mal}^{\prime}$ cev products play an important role in our efforts here, particularly in the context of the congruence $\nu$ on $\mathscr{L}(\mathscr{I})$. If $\mathscr{U}$ is a group variety and $\mathscr{V}$ is a combinatorial variety, then $\mathscr{U} \circ \mathscr{V}$ is the maximum variety in the $\nu$-class of $\mathscr{U} \vee \mathscr{V}$, where $\nu$ is the congruence on $\mathscr{L}(\mathscr{S})$ defined by $\mathscr{V}_{1} \vee \mathscr{V}_{2}$ if and only if $\mathscr{V}_{1} \cap \mathscr{G}=\mathscr{V}_{2} \cap \mathscr{G}$ and $\mathscr{V}_{1} \vee \mathscr{G}=\mathscr{V}_{2} \vee \mathscr{G}$, for all $\mathscr{V}_{1}, \mathscr{V}_{2} \in \mathscr{L}(\mathscr{I})$ (see, for e.g., [P; XII.2, XII.3]). By a result due to Reilly [R1], if $\mathscr{U}$ is a variety of groups and $\mathscr{V}$ is a combinatorial variety, then $[\mathscr{U} \vee \mathscr{V}, \mathscr{U} \circ \mathscr{V}]$ is the $\nu$-class of $\mathscr{V} \vee \mathscr{U}$. For further information on $\mathrm{Mal}^{\prime} c e v$ products we refer the reader to $[\mathbf{P}]$ or $[\mathbf{R 1}]$.

Define the binary operator $\mathrm{Wr}$ on the lattice of varieties of inverse semigroups by

$$
\operatorname{Wr}(\mathscr{U}, \mathscr{V})=\langle S \operatorname{wr}(T, I): S \in \mathscr{U} \text { and } T \in \mathscr{V}\rangle \quad(\mathscr{U}, \mathscr{V} \in \mathscr{L}(\mathscr{I})) .
$$


If $\mathscr{U}$ is a group variety and $\mathscr{V}$ is a variety of inverse semigroups then $\operatorname{Wr}(\mathscr{U}, \mathscr{V})=\mathscr{U} \circ \mathscr{V}($ see $[\mathbf{C}])$.

We find it convenient in our investigations to make use of the graphical representation of inverse semigroups introduced by Stephen [S], which he calls the Schützenberger representation of an inverse semigroup with presentation. Schützenberger graphs are defined as follows:

Let $P=(X ; R)$ be a fixed presentation of the inverse semigroup $S$ with $\tau$ the corresponding congruence on $F \mathscr{I}(X)$, the free inverse semigroup on $S$. Let $w \in S$ and $R_{w}$ the $\mathscr{R}$-class of $w$ in $S$. The Schützenberger graph of $R_{w}$ with respect to $P$ is the labelled digraph $\Gamma(w)$, where

$$
\begin{aligned}
& V(\Gamma(w))=R_{w}, \\
& E(\Gamma(w))=\left\{\left(v_{1}, x, v_{2}\right): v_{1}, v_{2} \in R_{w}, \quad x \in X \cup X^{-1}\right. \\
& \left.\quad \text { and } v_{1}(x \tau)=v_{2}\right\}
\end{aligned}
$$

The Schützenberger representation of $w$ (with respect to $P$ ) is the birooted labelled digraph $\left(w w^{-1}, \Gamma(w), w\right)$, where $w w^{-1}$ is the start vertex and $w$ is the end or terminal vertex. The Schützenberger representation of the semigroup $S$ is the family of birooted graphs $\left\{\left(w w^{-1}, \Gamma(w), w\right): w \in S\right\}$. Schützenberger graphs enjoy the following properties:

Let $v \in S, \Gamma(v)$ be its Schützenberger graph with respect to $P$, $v_{1}, v_{2}, v_{3} \in R_{v}$ and $w \in(X \cup X)^{+}$(see [S]).

(a) if $\left(v_{1}, x, v_{2}\right)$ is an edge in $\Gamma(v)$ then $\left(v_{2}, x^{-1}, v_{1}\right)$ is also an edge in $\Gamma(v)$;

(b) if $\left(v_{1}, x, v_{2}\right)$ and $\left(v_{1}, x, v_{3}\right)$ are edges in $\Gamma(v)$ then $v_{2}=v_{3}$;

(c) if $\left(v_{2}, x, v_{1}\right)$ and $\left(v_{3}, x, v_{1}\right)$ are edges in $\Gamma(v)$ then $v_{2}=v_{3}$;

(d) $v_{1}(w \tau)=v_{2}$ if and only if $w$ labels a $v_{1}-v_{2}$ walk;

(e) $(w \tau) \geq v$ if and only if $w$ labels an $e-v$ walk;

(f) $v_{1} \mathscr{D} v_{2}$ if and only if $\Gamma\left(v_{1}\right)$ is isomorphic to $\Gamma\left(v_{2}\right)$;

(g) $v_{1} \mathscr{R} v_{2}$ if and only if there exists an isomorphism from $\Gamma\left(v_{1}\right)$ to $\Gamma\left(v_{2}\right)$ such that $v_{1} v_{1}^{-1}$ is mapped to $v_{2} v_{2}^{-1}$;

(h) $v_{1} \mathscr{L} v_{2}$ if and only if there exists an isomorphism from $\Gamma\left(v_{1}\right)$ to $\Gamma\left(v_{2}\right)$ such that $v_{1}$ is mapped to $v_{2}$.

We will only be considering Schützenberger graphs of the $\mathscr{B}^{1}$-free inverse semigroup on (countably infinite) $X$ with respect to the presentation $P=\left(X ; \rho\left(\mathscr{B}^{1}\right)\right)$. For further properties and a detailed discussion of Schützenberger graphs we refer the reader to Stephen [S]. 
3. The variety $\mathscr{B}^{1}$. In this section we construct inverse semigroups which belong to the variety $\mathscr{B}^{1}$ which, in subsequent sections, will be used to construct inverse semigroups in $\operatorname{Wr}\left(\mathscr{U}, \mathscr{B}^{1}\right)$, where $\mathscr{U}$ is a variety of abelian groups. These semigroups will be used to define an infinite collection of varieties in the interval $\left[\mathscr{U} \vee \mathscr{B}^{1}, \mathrm{Wr}\left(\mathscr{U}, \mathscr{B}^{1}\right)\right]$. Throughout the remainder of this note $\rho$ will denote the fully invariant congruence on $F \mathscr{F}(X)$ corresponding to $\mathscr{B}^{1}$.

Before we proceed, we require some notation. For any word $w \in$ $X \cup X^{-1}$, denote by $w_{A}$ the word obtained from $w$ by deleting all occurrences of variables not in $A$. For example, $\left(x_{1} x_{2} x_{1}^{-1} x_{3} x_{2} x_{1}\right)_{\left\{x_{1}\right\}}$ is the word $x_{1} x_{1}^{-1} x_{1}$.

LemMA 3.1. Let $w$ and $v$ be words over $X \cup X^{-1}$. Then $w \rho v$ if and only if $c(w)=c(v)$ and for all $A \subseteq c(w), A \neq \varnothing, w_{A} \rho(\mathscr{B}) v_{A}$.

Proof. $w \rho v$ if and only if $B_{2}^{1}$ satisfies the equation $w=v$. Since $B_{2}^{1}$ possesses an identity, $B_{2}^{1}$ satisfies the equation $w=v$ if and only if $B_{2}$ satisfies $w_{A}=v_{A}$ for all $A \subseteq c\left(w_{A}\right)=c\left(v_{A}\right)$. This is equivalent to $c(w)=c(v)$ and for all $A \subseteq c(w), A \neq \varnothing, w_{A} \rho(\mathscr{B}) v_{A}$.

Corollary 3.2. Let $w$ and $v$ be words over $X \cup X^{-1}$. Then $w \rho v$ if and only if $c(w)=c(v)$ and for all $A \subseteq c(w), A \neq \varnothing, w_{A} \rho v_{A}$.

Proof. If $w \rho v$ then by Lemma 3.1, $c(w)=c(v)$ and for all $A \subseteq c(w), A \neq \varnothing, w_{A} \rho(\mathscr{B}) v_{A}$. But then for any $A \subseteq c(w)=c(v)$, for all $B \subseteq A, B \neq \varnothing, w_{B} \rho(\mathscr{B}) v_{B}$ and so by Lemma 3.1, $w_{A} \rho v_{A}$. On the other hand, if $c(w)=c(v)$ and for all $A \subseteq c(w), A \neq \varnothing$, $w_{A} \rho v_{A}$, then in particular, $w=w_{c(w)} \rho v_{c(w)}=v_{c(v)}=v$.

LEMMA 3.3. If $S \in \mathscr{B}^{1}$ then $S^{1} \in \mathscr{B}^{1}$.

Proof. Suppose that $\mathscr{B}^{1}$ satisfies the equation $w=v$, where $c(w)$ $=c(v)=\left\{x_{1}, \ldots, x_{n}\right\}$. Let $s_{1}, \ldots, s_{n}$ be arbitrarily chosen elements of $S^{1}$ with repetitions allowed. Suppose that $s_{i_{1}}, \ldots, s_{i_{k}}$ are those $s_{i}$ that are the identity of $S^{1}$. Then $S^{1}$ satisfies $w\left[s_{1}, \ldots, s_{n}\right]=$ $v\left[s_{1}, \ldots, s_{n}\right]$ if $S$ satisfies $w_{A}\left[s_{1}, \ldots, s_{n}\right]=v_{A}\left[s_{1}, \ldots, s_{n}\right]$, where $A=\left\{x_{1}, \ldots, x_{n}\right\} \backslash\left\{x_{i_{1}}, \ldots, x_{i_{k}}\right\}$. Since $S \in \mathscr{B}^{1}, S$ does satisfy $w_{A}\left[s_{1}, \ldots, s_{n}\right]=v_{A}\left[s_{1}, \ldots, s_{n}\right]$ by Corollary 3.2 and so, as a result, $w\left[s_{1}, \ldots, s_{n}\right]=v\left[s_{1}, \ldots, s_{n}\right]$ is true in $S^{1}$. Since the $s_{i}$ were chosen arbitrarily, $S^{1}$ satisfies the equation $w=v$. Therefore, $S^{1} \in \mathscr{B}^{1}$.

We require some further notation for this section. Let $w \in$ $\left(X \cup X^{-1}\right)^{+}$. We write $w \equiv v$ to mean $w$ and $v$ are identical words, 
letter for letter, over a common alphabet (in this case $X \cup X^{-1}$ ). We say that the word $v$ is a cyclic shift of $w$ if $w \equiv u_{1} u_{2}$ and $v \equiv u_{2} u_{1}$ for words $u_{1}, u_{2}$ over the alphabet of $w$. For each $n \in \omega$, we denote by $\tau_{n}$ the equation $x_{1} x_{2} \cdots x_{n} x_{1}^{-1} x_{2}^{-1} \cdots x_{n}^{-1} \in E$. Observe that if $w$ is the word $x_{1} x_{2} \cdots x_{n} x_{1}^{-1} x_{2}^{-1} \cdots x_{n}^{-1}$ then any cyclic shift of $w$ can be written $y_{1} y_{2} \cdots y_{n} y_{1}^{-1} y_{2}^{-1} \cdots y_{n}^{-1}$ (where the $y_{i}$ all belong to $\left.\left\{x_{1}, \ldots, x_{n}, x_{1}^{-1}, \ldots, x_{n}^{-1}\right\}\right)$.

The remainder of this section is devoted to a construction of a family of inverse semigroups $\left\{S\left(\tau_{n}\right): n \in \omega\right\}$ each of which belongs to the variety $\mathscr{B}^{-1}$. For each $n \in \omega, S\left(\tau_{n}\right)$ is obtained from the $\mathscr{B}^{1}$-free inverse semigroup by first identifying the ideal consisting of those elements whose $\mathscr{D}$-class does not lie above the $\mathscr{D}$-class of $x_{1} x_{2} \cdots x_{n} x_{1}^{-1} x_{2}^{-1} \cdots x_{n}^{-1} \rho$ (which results in an ideal extension of the principal factor of the $\mathscr{D}$-class of $x_{1} x_{2} \cdots x_{n} x_{1}^{-1} x_{2}^{-1} \cdots x_{n}^{-1} \rho$, a Brandt semigroup) and then mapping this semigroup into the translational hull of the principal factor corresponding to the $\mathscr{D}$-class of $x_{1} x_{2} \cdots x_{n} x_{1}^{-1} x_{2}^{-1} \rho$. In order to do this we require some knowledge of the $\mathscr{D}$-class of $x_{1} x_{2} \cdots x_{n} x_{1}^{-1} x_{2}^{-1} \cdots x_{n}^{-1} \rho$.

LEMMA 3.4. Let $w=x_{1} x_{2} \cdots x_{n} x_{1}^{-1} x_{2}^{-1} \cdots x_{n}^{-1}$ and suppose that $v=y_{1} y_{2} \cdots y_{n} y_{1}^{-1} y_{2}^{-1} \cdots y_{n}^{-1}$ is a cyclic shift of $w$. Let $a \in X \cup X^{-1}$.

(a) $v \rho$ is an idempotent;

(b) $(v a \rho) \mathscr{R}(v \rho)$ if and only if $a=y_{1}$ or $a=y_{n}$.

Proof. (a) As we remarked in $\S 2, \mathscr{B}^{1}$ is contained in $\mathscr{A}_{2}^{\max }$ (because it has $E$-unitary covers over the variety $\mathscr{A}_{2}$ of abelian groups of exponent two; see [PR]). Since $\mathscr{A}_{2}$ satisfies the equation $v=v^{2}$, $\mathscr{A}_{2}^{\max }$ and hence $\mathscr{B}^{1}$ satisfies $v=v^{2}$. Thus, $v \rho$ is an idempotent.

(b) Since $v \rho$ is an idempotent, if $a=y_{1}$ or $a=y_{n}$ then $(v a \rho) \mathscr{R}(v \rho)$. On the other hand, suppose that $(v a \rho) \mathscr{R}(v \rho)$. Then $v a a^{-1} v^{-1} \rho v v^{-1}$ and so $c(v a)=c(v)$. Thus, $a \in c(v)$. But $(v a \rho) \mathscr{R}(v \rho)$ also implies that $v a a^{-1} \rho v$. If $a=y_{i}^{-1}$ for some $i$, then $\left(v a a^{-1}\right)_{\left\{y_{l}\right\}}=y_{i} y_{i}^{-1} y_{i}^{-1} y_{i} \rho(\mathscr{B}) y_{i}^{2}$, while $v_{\left\{y_{l}\right\}}=y_{i} y_{i}^{-1} \phi(\mathscr{B}) y_{i}^{2}$ and so, by Lemma 3.2, $v a a^{-1} \not v$. Therefore, $a=y_{i}$ for some $i$. If $1<i<n$ then $\left(v_{a a}^{-1}\right)_{\left\{y_{1}, y_{l}, y_{n}\right\}}=y_{1} y_{i} y_{n} y_{1}^{-1} y_{i}^{-1} y_{n}^{-1} y_{i} y_{i}^{-1}$ and $v_{\left\{y_{1}, y_{l}, y_{n}\right\}}=y_{1} y_{i} y_{n} y_{1}^{-1} y_{i}^{-1} y_{n}^{-1}$. If $b$ is any non-idempotent element of $B_{2}$, then substituting $b$ for $y_{1}$ and $y_{n}$ and substituting $b^{-1}$ for $y_{i}$, yields that $\left(v a a^{-1}\right)_{\left\{y_{1}, y_{l}, y_{n}\right\}} \not(\mathscr{B}) v_{\left\{y_{1}, y_{l}, y_{n}\right\}}$. As a consequence, $y_{i}$ must be either $y_{1}$ or $y_{n}$. 
LEMMA 3.5. Let $w=x_{1} x_{2} \cdots x_{n} x_{1}^{-1} x_{2}^{-1} \cdots x_{n}^{-1}$ and suppose that $u$ is a proper initial segment of $w$ with $w \equiv u u^{\prime}$. Let $a \in X \cup X^{-1}$.

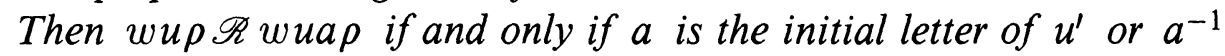
is the terminal letter of $u$ in the case that $u$ is not the empty word, and in the case that $u$ is the empty word, $a$ is the initial letter of $u^{\prime}$ or $a^{-1}$ is the terminal letter of $u^{\prime}$.

Proof. If $u$ is the empty word then the statement follows immediately from Lemma 3.4 , so assume that $u$ is not the empty word.

First suppose that $w u \rho \mathscr{R} w u a \rho$. Then $w u \rho=u u^{\prime} u \rho \mathscr{L} u^{\prime} u \rho$ since $u^{\prime} u$ is a cyclic shift of $w$ and any cyclic shift of $w$ is an idempotent modulo $\rho$. Therefore, wu $\mathscr{R} w u a \rho$ implies that $u^{\prime} u \rho \mathscr{R} u^{\prime} u a \rho$ (this follows from the more general result that $t \mathscr{L} s$ implies that $t \mathscr{R} t a$ if and only if $s \mathscr{R} s a$ ). Since $u^{\prime} u$ is a cyclic shift of $w$, we have by Lemma 3.4 that $a$ is either the initial letter of $u^{\prime}$ or $a^{-1}$ is the terminal letter of $u$.

For the converse, first note that if $a$ is the initial letter of $u^{\prime}$ then $u a$ is an initial segment of $w$ and so, since $w \rho$ is an idem-

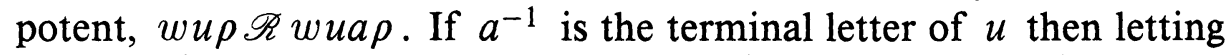
$u \equiv u^{*} a^{-1}$ we obtain that $w u a \equiv w u^{*} a^{-1} a \equiv u^{*} a^{-1} u^{\prime} u^{*} a^{-1} a$. Since $a^{-1} u^{\prime} u^{*}$ is a cyclic shift of $w, a^{-1} u^{\prime} u^{*} \rho$ is an idempotent by Lemma $3.4(\mathrm{a})$ and as a result,

$$
\begin{aligned}
w u a & \equiv w u^{*} a^{-1} a \equiv u^{*} a^{-1} u^{\prime} u^{*} a^{-1} a \rho u^{*} a^{-1} a a^{-1} u^{\prime} u^{*} \rho u^{*} a^{-1} u^{\prime} u^{*} \\
& \equiv u u^{\prime} u^{*} \equiv w u^{*} .
\end{aligned}
$$

It is now immediate that $w u \rho \mathscr{R} w u^{*} \rho=w u a \rho$.

LEMMA 3.6. Let $w=x_{1} x_{2} \cdots x_{n} x_{1}^{-1} x_{2}^{-1} \cdots x_{n}^{-1}$. For any word $v$ over $X \cup X^{-1}, w \rho \mathscr{R} v \rho$ if and only if $v \rho w u$ for some initial segment $u$ of $w$.

Proof. Suppose that $w \rho \mathscr{R} v \rho$, say $w a_{1} \cdots a_{k} \rho v$, where $a_{1}, \ldots$, $a_{k} \in X \cup X^{-1}$. We prove by induction on $k$ that $w a_{1} \cdots a_{k} \rho \mathscr{R} w \rho$ implies that $w a_{1} \cdots a_{k} \rho w u$ for some initial segment $u$ of $w$. If $k=1$ then $w a_{1} \rho \mathscr{R} w \rho$ implies by Lemma 3.4 that $a_{1}=x_{1}$ or $x_{n}$. If $a=x_{1}$ then $a_{1}$ is an initial segment of $w$ already. If $a_{1}=x_{n}$ then $w a_{1} \rho w w x_{n}$. Now

$$
\begin{aligned}
w w x_{n} \equiv & x_{1} \cdots x_{n} x_{1}^{-1} \cdots x_{n-1}^{-1}\left[x_{n}^{-1} x_{1} \cdots x_{n} x_{1}^{-1} \cdots x_{n-1}^{-1}\right] x_{n}^{-1} x_{n} \\
& \rho x_{1} \cdots x_{n} x_{1}^{-1} \cdots x_{n-1}^{-1}\left[x_{n}^{-1} x_{1} \cdots x_{n} x_{1}^{-1} \cdots x_{n-1}^{-1}\right]
\end{aligned}
$$


since $\left[x_{n}^{-1} x_{1} \cdots x_{n} x_{1}^{-1} \cdots x_{n-1}^{-1}\right]$ is a cyclic shift of $w$ and so $\left[x_{n}^{-1} x_{1} \cdots x_{n} x_{1}^{-1} \cdots x_{n-1}^{-1}\right] \rho$ is an idempotent.

But

$x_{1} \cdots x_{n} x_{1}^{-1} \cdots x_{n-1}^{-1}\left[x_{n}^{-1} x_{1} \cdots x_{n} x_{1}^{-1} \cdots x_{n-1}^{-1}\right] \equiv w x_{1} \cdots x_{n} x_{1}^{-1} \cdots x_{n-1}^{-1}$

and so as a consequence, $v \rho w x_{1} \cdots x_{n} x_{1}^{-1} \cdots x_{n-1}^{-1}$.

Now suppose that $k>1 . \quad w a_{1} \cdots a_{k} \rho \mathscr{R} w \rho$ implies that $w \rho \mathscr{R} w a_{1} \cdots a_{k-1} \rho$ and so, by the induction hypothesis, $w a_{1} \cdots$ $a_{k-1} \rho w u$ for some initial segment $u$ of $w \equiv u u^{\prime}$. If $u$ is the empty word, then $w a_{1} \cdots a_{k} \rho w a_{k} \mathscr{R} w \rho$ and this is the same as the case

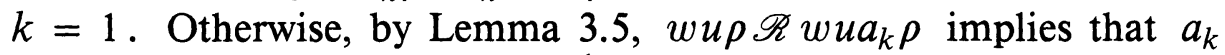
is the initial letter of $u^{\prime}$ or $a_{k}^{-1}$ is the terminal letter of $u$. If $a$ is the initial letter of $u^{\prime}$ then $v \rho w a_{1} \cdots a_{k} \rho w u a_{k}$ and $u a_{k}$ is an initial segment of $w$. If $a_{k}^{-1}$ is the terminal letter of $u$ then setting $u \equiv b_{1} \cdots b_{m}$ we obtain that $v \rho w a_{1} \cdots a_{k} \rho w u a_{k}$ and

$$
\begin{aligned}
w u a_{k} \equiv & w b_{1} \cdots b_{m} b_{m}^{-1} \\
& \equiv b_{1} \cdots b_{m-1}\left[b_{m} u^{\prime} b_{1} \cdots b_{m-1}\right] b_{m} b_{m}^{-1} \\
& \rho b_{1} \cdots b_{m-1}\left[b_{m} u^{\prime} b_{1} \cdots b_{m-1}\right]
\end{aligned}
$$

since $\left[b_{m} u^{\prime} b_{1} \cdots b_{m-1}\right]$ is a cyclic shift of $w$ and so must ry an idempotent modulo $\rho$. But $b_{1} \cdots b_{m-1}\left[b_{m} u^{\prime} b_{1} \cdots b_{m-1}\right] \equiv w b_{1} \cdots b_{m-1}$ and so $v \rho w b_{1} \cdots b_{m-1}$ and $b_{1} \cdots b_{m-1}$ is an initial segment of $w$.

Since $w \rho$ is an idempotent, the converse is immediate.

Schützenberger graphs provide a concise, visual representation of a $\mathscr{D}$-class. Because of this, in the following theorem we describe the $\mathscr{D}$-classes of the words $\left\{x_{1} x_{2} \cdots x_{n} x_{1}^{-1} x_{2}^{-1}: n \in \omega, n>1\right\}$ relative to the variety $\mathscr{B}^{1}$ in this way.

THEOREM 3.7. Let $w=x_{1} x_{2} \cdots x_{n} x_{1}^{-1} x_{2}^{-1} \cdots x_{n}^{-1}$. The following graph is isomorphic to the Schützenberger graph of $w$ relative to $\mathscr{B}^{1}$, where $v_{1}$ is both the start and end vertex.

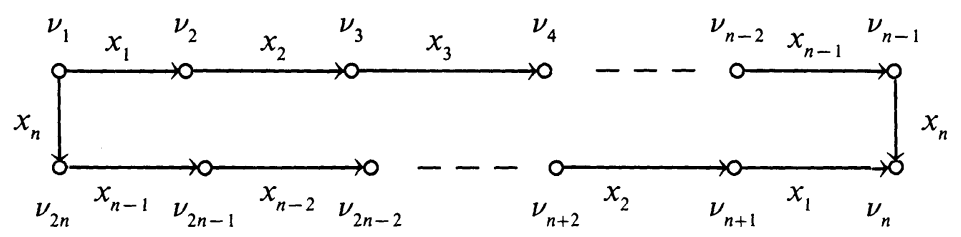

FIGURE 3.1

The Schützenberger graph of $w=x_{1} x_{2} \cdots x_{n} x_{1}^{-1} x_{2}^{-1} \cdots x_{n}^{-1}$ with respect to $\mathscr{B}^{1}$. 
Proof. By Lemma 3.6 there are at most $2 n$ vertices in the Schützenberger graph $\Gamma$ of $w$ relative to $\mathscr{B}^{1}$ as there are $2 n$ initial segments of $w$ not identical to $w$. It is a simple exercise to verify, using Lemma 3.1, that if $u$ and $u^{\prime}$ are two proper initial segments of $w$ (that is, neither $u$ nor $u^{\prime}$ is identical to $w$ ) then $w u \rho w u^{\prime}$ implies that $u \equiv u^{\prime}$. By Lemma 3.5, $\left(w u_{1} \rho, x, w u_{2} \rho\right)$ is an edge of $\Gamma$ if and only if $x^{-1}$ is the terminal letter of $u_{1}$ or $x$ is the initial letter of $u_{1}^{\prime}$, where $u_{1} u_{1}^{\prime} \equiv w$. If $x$ is the initial letter of $u_{1}^{\prime}$, then $w u_{2}$ and $w u_{1} x$ are $\rho$-equivalent with both $u_{1} x$ and $u_{2}$ initial segments of $w$. Thus, $u_{1} x \equiv u_{2}$. If $x^{-1}$ is the terminal letter of $u_{1}$ then writing $u_{1} \equiv$ $u_{1}^{*} x^{-1}$ we have $w u_{1}^{*} x^{-1} x \rho w u_{2}$. Since $w u_{1}^{*} \rho \mathscr{R} w u_{1} \equiv w u_{1}^{*} x^{-1} \rho$, we have that $w u_{1}^{*} \rho w u_{1}^{*} x^{-1} x \rho w u_{2}$. Since both $u_{1}^{*}$ and $u_{2}$ are initial segments of $w, w u_{1}^{*} \equiv w u_{2}$ and so $w u_{2} x^{-1} \equiv w u_{1}$. Finally, if $u_{1}$ is the empty word and $x^{-1}$ is the terminal letter of $w$ then $x^{-1}$ is the terminal letter of $w w \equiv w w^{*} x^{-1} \rho w$ and $w w^{*} x^{-1} x \rho w u_{2}$. But, $w w^{*} x^{-1} x \rho w w^{*}$ and both $w^{*}$ and $u_{2}$ are initial segments of $w$, so $w u_{2} \equiv w w^{*}$, whence $w u_{2} x^{-1} \equiv w w$.

It follows from these remarks that $\Gamma$ is isomorphic to the graph described above via the map which sends $w u \rho$ to $v_{|u|+1}$, for all proper initial segments $u$ of $w$.

Definition 3.8. Let $F$ be the $\mathscr{B}^{1}$-free inverse semigroup on $X=$ $\left\{x_{i}: i \in \omega\right\}$. Let $w_{n}$ be the word $x_{1} \cdots x_{n} x_{1}^{-1} \cdots x_{n}^{-1}$ for each $n \in$ $\omega$. Denote the ideal $\left\{v \in F: J_{v} ¥ J_{w_{n} \rho}\right\}$ of $F$ by $I\left(\tau_{n}\right)$ and let $J\left(\tau_{n}\right)=F / I\left(\tau_{n}\right)$. Now $J\left(\tau_{n}\right)$ is an ideal extension of $J_{w_{n} \rho}^{0}$ which is isomorphic to $B(\{1\}, 2 n)$. Let $S\left(\tau_{n}\right)$ be the image of $J\left(\tau_{n}\right)$ under the canonical homomorphism into the translational hull $\Omega\left(J_{w_{n} \rho}^{0}\right)$ of $J_{w_{n} \rho}^{0}$.

LEMMA 3.9. The semigroups $S\left(\tau_{n}\right)$ and $S\left(\tau_{n}\right)^{1}$ belong to $\mathscr{B}^{1}$, for all $n \in \omega, n \geq 2$.

Proof. The semigroup $S\left(\tau_{n}\right)$ is a homomorphic image of the $\mathscr{B}^{1}$ free inverse semigroup on $X$ and so is an element of $\mathscr{B}^{1}$. The semigroup $S\left(\tau_{n}\right)^{1} \in \mathscr{B}^{1}$ by Lemma 3.3.

In the following section we will use the $S\left(\tau_{n}\right)$ to construct a family of inverse semigroups which belong to $\mathrm{Wr}\left(\mathscr{A}_{m}, \mathscr{B}^{1}\right)$ but not to $\mathscr{A}_{m} \mathrm{~V}$ $\mathscr{B}^{1}$, for $m \in \omega$. Before we do so, we describe the $S\left(\tau_{n}\right)$.

The inverse semigroup $S\left(\tau_{n}\right)$ is isomorphic to the Wagner representation of the $\mathscr{B}^{1}$-free inverse semigroup on $X$ restricted to $R_{w_{n} \rho}$. 
That is, if $\alpha_{w}$ is the element of $\mathscr{I}\left(F \mathscr{B}^{1}(X)\right)$ corresponding to $w \rho$ in the Wagner representation of $F \mathscr{B}^{1}(X)$, then in the restricted (to $\left.R_{w_{n} \rho}\right)$ Wagner representation, $\alpha_{w}^{\prime}$ corresponds to $w \rho$, where $\mathbf{d} \alpha_{w}^{\prime}=$ $\left\{u \rho \in \mathbf{d} \alpha_{w}: u \rho \mathscr{R} w_{n} \rho\right.$ and $\left.(u \rho) \alpha_{w} \mathscr{R} w_{n} \rho\right\}$ and for all $u \rho \in \mathbf{d} \alpha_{w}^{\prime}$, $(u \rho) \alpha_{w}^{\prime}=(u \rho) \alpha_{w}$.

An added advantage to using the Schützenberger graph description in Theorem 3.7 is that we can read directly from the graph the image of any word of $J\left(\tau_{n}\right)$ under the canonical homomorphism into $\Omega\left(J_{w_{n} \rho}^{0}\right) \cong \mathscr{I}\left(R_{w_{n} \rho} \rho\right)$. The inverse semigroup $S\left(\tau_{n}\right)$ is generated by the image of the $x_{i}$ under the canonical homomorphism and, for each $i=1, \ldots, n$, the domain of the image of $x_{i}$ is the set of vertices $v$ for which there is an edge labelled by $x_{i}$ starting at $v$ and $v$ is mapped to the terminal vertex of that edge. It is straightforward to verify that $S\left(\tau_{n}\right)$ is (isomorphic to) the inverse subsemigroup of $\mathscr{F}\left(R_{w_{n}} \rho\right)$ generated by $\left\{\alpha_{i}: i=1, \ldots, n\right\}$ where for each $i$,

$$
\mathbf{d} \alpha_{i}=\left\{w_{n} x_{1} \cdots x_{i-1} \rho, w_{n} x_{1} \cdots x_{n} x_{1}^{-1} \cdots x_{i}^{-1} \rho\right\}
$$

and

$$
\begin{gathered}
w_{n} x_{1} \cdots x_{i-1} \rho \alpha_{i}=w_{n} x_{1} \cdots x_{i} \rho \\
w_{n} x_{1} \cdots x_{n} x_{1}^{-1} \cdots x_{i}^{-1} \rho \alpha_{i} \\
=w_{n} x_{1} \cdots x_{n} x_{1}^{-1} \cdots x_{i}^{-1} x_{i} \rho w_{n} x_{1} \cdots x_{n} x_{1}^{-1} \cdots x_{i-1}^{-1} .
\end{gathered}
$$

4. Inverse semigroups in $\operatorname{Wr}\left(\mathscr{A}_{m}, \mathscr{B}^{1}\right)$. The semigroups constructed in $\S 3$ can be used to construct semigroups in $\operatorname{Wr}\left(\mathscr{A}_{m}, \mathscr{B}^{1}\right)$ for $m \in \omega$. Since $S\left(\tau_{n}\right)$ is isomorphic to the Wagner representation of $F \mathscr{B}^{1}(X)$ restricted to $R_{w_{n} \rho}$, it can be represented as an inverse subsemigroup of $\mathscr{I}\left(R_{w_{n} \rho}\right)$ for all $n \in \omega$. Thus, for any group $G$ belonging to $\mathscr{A}_{m}, m \in \omega, G \operatorname{wr}\left(S\left(\tau_{n}\right), R_{w_{n}}\right) \in \mathrm{Wr}\left(\mathscr{A}_{m}, \mathscr{B}^{1}\right)$. The semigroups we construct in this section are inverse subsemigroups of semigroups of this form and so belong to $\mathrm{Wr}\left(\mathscr{A}_{m}, \mathscr{B}^{1}\right)$.

For each $n \in \omega, n \geq 2$, let $C_{n}$ denote the cyclic group of order $n$.

Definition 4.1. Let $m, n \in \omega, m, n \geq 2$. Let 1 denote the identity of $C_{m}$ and let $g$ be a generator of $C_{m}$. Let

$$
A_{m, n} \subseteq C_{m} \operatorname{wr}\left(S\left(\tau_{n}\right), R_{w_{n}}\right)
$$

be defined as follows:

Let $\left\{\alpha_{i}: i=1, \ldots, n\right\}$ be the generators of $S\left(\tau_{n}\right)$ as described at the end of the previous section. For $i=1, \ldots, n-1$, define the map $\phi_{i}$ from $R_{w_{n}}$ into $C_{m}$ by setting

$$
\mathbf{d} \phi_{i}=\mathbf{d} \alpha_{i}=\left\{w_{n} x_{1} \cdots x_{i-1} \rho, w_{n} x_{1} \cdots x_{n} x_{1}^{-1} \cdots x_{i}^{-1} \rho\right\}
$$


and defining $\left(w_{n} x_{1} \cdots x_{i-1} \rho\right) \phi_{i}=1,\left(w_{n} x_{1} \cdots x_{n} x_{1}^{-1} \cdots x_{i}^{-1} \rho\right) \phi_{i}=1$. Define the map $\phi_{n}$ from $R_{w_{n}}$ into $C_{m}$ by setting $\mathbf{d} \phi_{n}=\mathbf{d} \alpha_{n}=$ $\left\{w_{n} x_{1} \cdots x_{n-1} \rho, w_{n} \rho\right\}$ and defining $\left(w_{n} x_{1} \cdots x_{n-1} \rho\right) \phi_{n}=1,\left(w_{n} \rho\right) \phi_{n}$ $=g$. Then $\left(\phi_{i}, \alpha_{i}\right) \in C_{m} \operatorname{wr}\left(S\left(\tau_{n}\right), R_{w_{n}}\right)$ for $i=1, \ldots, n$.

Let

$$
\begin{aligned}
A_{m, n}= & \left\{(\psi, \beta) \in C_{m} \operatorname{wr}\left(S\left(\tau_{n}\right), R_{w_{n}}\right):|\mathbf{d} \psi|=|\mathbf{d} \beta| \leq 1\right\} \\
& \cup\left\{\left(\phi_{i}, \alpha_{i}\right): i=1, \ldots, n\right\} .
\end{aligned}
$$

Define $T_{m, n}$ to be the inverse subsemigroup of $C_{m} \operatorname{wr}\left(S\left(\tau_{n}\right), R_{w_{n}}\right)$ generated by $A_{m, n}$. Observe that $T_{m, n}$ is an ideal extension of a Brandt semigroup over the group $C_{m}$. It is not difficult to see that $T_{m, n}$ is in fact the following:

$$
\begin{array}{r}
\left\{(\psi, \beta) \in C_{m} \operatorname{wr}\left(S\left(\tau_{n}\right), R_{w_{n}}\right):|\mathbf{d} \psi|=|\mathbf{d} \beta| \leq 1\right\} \\
\cup\left\{\left(\phi_{i}, \alpha_{i}\right),\left(\phi_{i}, \alpha_{i}\right)^{-1},\left(\phi_{i}, \alpha_{i}\right)\left(\phi_{i}, \alpha_{i}\right)^{-1},\right. \\
\left.\left(\phi_{i}, \alpha_{i}\right)^{-1}\left(\phi_{i}, \alpha_{i}\right): i=1, \ldots, n\right\} .
\end{array}
$$

LEMMA 4.2. For each $m, n \in \omega, m, n \geq 2$,

(a) $T_{m, n} \in \mathrm{Wr}\left(\mathscr{A}_{m}, \mathscr{B}^{1}\right)$ but $T_{m, n} \notin \mathscr{B}^{1}$;

(b) $T_{m, n}^{1} \in \mathrm{Wr}\left(\mathscr{A}_{m}, \mathscr{B}^{1}\right)$ but $T_{m, n}^{1} \notin \mathscr{B}^{1}$;

(c) $\mathscr{A}_{m} \vee \mathscr{B}^{1} \subseteq\left\langle T_{m, n}\right\rangle \subseteq \mathrm{Wr}\left(\mathscr{A}_{m}, \mathscr{B}^{1}\right)$;

(d) $\mathscr{A}_{m} \vee \mathscr{B}^{1} \subseteq\left\langle T_{m, n}^{1}\right\rangle \subseteq \mathrm{Wr}\left(\mathscr{A}_{m}, \mathscr{B}^{1}\right)$.

Proof. $T_{m, n}^{1}$ is an inverse subsemigroup of $C_{m} \operatorname{wr}\left(S\left(\tau_{n}\right)^{1}, R_{w_{n}}\right)$ and $S\left(\tau_{n}\right)^{1} \in \mathscr{B}^{1}$ by Lemma 3.9. Thus, $T_{m, n}^{1} \in \mathrm{Wr}\left(\mathscr{A}_{m}, \mathscr{B}^{1}\right)$ by the definition of the Wr operator. As a consequence, $T_{m, n} \in \mathrm{Wr}\left(\mathscr{A}_{m}, \mathscr{B}^{1}\right)$ since $T_{m, n}$ is an inverse subsemigroup of $T_{m, n}^{1}$. On the other hand, $T_{m, n}$ is an ideal extension of a Brandt semigroup over $C_{m}$ and so contains a subgroup isomorphic to $C_{m}$. Thus, $T_{m, n} \notin \mathscr{B}^{1}$ since $\mathscr{B}^{1}$ is a combinatorial variety. Since $T_{m, n}$ is an inverse subsemigroup of $T_{m, n}^{1}$ we also have that $T_{m, n}^{1} \notin \mathscr{B}^{1}$. This proves both (a) and (b).

Both $T_{m, n}^{1}$ and $T_{m, n}$ contain subgroups isomorphic to $C_{m}$ and so $\mathscr{A}_{m} \subseteq\left\langle T_{m, n}^{1}\right\rangle$ and $\mathscr{A}_{m} \subseteq\left\langle T_{m, n}\right\rangle$ since $\mathscr{A}_{m}$ is generated by $C_{m}$. The natural homomorphism onto the second coordinate maps $T_{m, n}$ onto an inverse semigroup isomorphic to $S\left(\tau_{n}\right) \in \mathscr{B}^{1}$, and maps $T_{m, n}^{1}$ onto an inverse semigroup isomorphic to $S\left(\tau_{n}\right)^{1} \in \mathscr{B}^{1}$. Since both $S\left(\tau_{n}\right)$ and $S\left(\tau_{n}\right)^{1}$ contain copies of $B_{2}^{1}$, it follows that $\mathscr{B}^{1} \subseteq\left\langle T_{m, n}^{1}\right\rangle$ and $\mathscr{B}^{1} \subseteq\left\langle T_{m, n}\right\rangle$. Consequently, we have that $\mathscr{A}_{m} \vee \mathscr{B}^{1} \subseteq\left\langle T_{m, n}\right\rangle$ and $\mathscr{A}_{m} \vee \mathscr{B}^{1} \subseteq\left\langle T_{m, n}^{1}\right\rangle$. It is immediate from parts (a) and (b) that $\left\langle T_{m, n}\right\rangle \subseteq \mathrm{Wr}\left(\mathscr{A}_{m}, \mathscr{B}^{1}\right)$ and $\left\langle T_{m, n}^{1}\right\rangle \subseteq \mathrm{Wr}\left(\mathscr{A}_{m}, \mathscr{B}^{1}\right)$. This completes the proofs of (c) and (d). 
Lemma 4.3. Let $m, n \in \omega, m, n \geq 2$. Neither $T_{m, n}$ nor $T_{m, n}^{1}$ satisfies the equation $\tau_{n}$.

Proof. Substitute $\left(\phi_{i}, \alpha_{i}\right)$ for $x_{i}, i=1, \ldots, n$.

In the following lemma we use the term kernel to mean the minimum nonzero ideal of an inverse semigroup, if it exists.

LEMma 4.4. Let $m, n \in \omega, m, n \geq 2 . T_{m, n}$ satisfies the equation $\tau_{k}$ for $k<n$.

Proof. Towards a contradiction, suppose that $T_{m, n}$ does not satisfy $\tau_{k}$ for some $k<n$. Assume that $k$ is the least such integer and let $\left(\psi_{1}, \beta_{1}\right), \ldots,\left(\psi_{k}, \beta_{k}\right) \in T_{m, n}$ be such that

$$
x_{1} \cdots x_{k} x_{1}^{-1} \cdots x_{k}^{-1}\left[\left(\psi_{1}, \beta_{1}\right), \ldots,\left(\psi_{k}, \beta_{k}\right)\right]=(\psi, \beta)
$$

is not an idempotent in $T_{m, n}$.

We first make a few observations.

(i) $|\mathbf{d} \beta|=1$ : If $|\mathbf{d} \beta|=0$ then we immediately have that $(\psi, \beta)$ is an idempotent. If $|\mathbf{d} \beta|=2$ then the $\left(\psi_{i}, \beta_{i}\right)$ all belong to the same $\mathscr{D}$-class, namely, the $\mathscr{D}$-class $D$ of $(\psi, \beta)$. [This is because $T_{m, n}$ is completely semisimple and so $\mathscr{D}=\mathscr{J}$. Thus, the $\mathscr{D}$-class of $(\psi, \beta)$ is contained in the $\mathscr{D}$-class of $\left(\psi_{i}, \beta_{i}\right)$ for all $i$. But if $|\mathbf{d} \beta|=2$, then the $\mathscr{D}$-class of $(\psi, \beta)$ is a maximal $\mathscr{D}$-class in $T_{m, n}$ and so $(\psi, \beta)$ is $\mathscr{D}$-related to $\left(\psi_{i}, \beta_{i}\right)$ for all $i$.] But $D^{0}$ is a Brandt semigroup and as such satisfies $\tau_{k}$. Since $x_{1} \cdots x_{k} x_{1}^{-1} \cdots x_{k}^{-1}\left[\left(\psi_{1}, \beta_{1}\right), \ldots,\left(\psi_{k}, \beta_{k}\right)\right]$ $=(\psi, \beta)$ in $D^{0}$ and $(\psi, \beta) \neq 0$, we conclude that, in this case, $(\psi, \beta)$ is an idempotent. The only remaining possibility is that $|\mathbf{d} \beta|=1$.

(ii) If $\mathbf{d} \beta=\{v\}$ then $v \beta=v$ and $v \psi$ is not an idempotent. We know that $\beta$ is an idempotent of $\left(S\left(\tau_{n}\right), R_{w_{n}}\right)$ since the natural homomorphism of $T_{m, n}$ onto its second coordinate has image $S\left(\tau_{n}\right)$ which, by Lemma 3.9 , is a member of $\mathscr{B}^{1}$ and $\mathscr{B}^{1}$ satisfies the equation $\tau_{k}$. Thus, $v \beta=v$. Also, $v \psi$ is not an idempotent lest $(\psi, \beta)=(\psi, \beta)^{2}$.

(iii) If $(\psi, \beta)$ is not an idempotent then for any cyclic shift $y_{1} \cdots y_{n} y_{1}^{-1} \cdots y_{n}^{-1}$ of $x_{1} \cdots x_{k} x_{1}^{-1} \cdots x_{k}^{-1}$ we have that $y_{1} \cdots y_{n} y_{1}^{-1} \cdots$ $y_{n}^{-1}\left[\left(\psi_{1}, \beta_{1}\right), \ldots,\left(\psi_{k}, \beta_{k}\right)\right]$ is not an idempotent. To see this note that if $y_{1} \cdots y_{n} y_{1}^{-1} \cdots y_{n}^{-1}$ is a cyclic shift of $x_{1} \cdots x_{k} x_{1}^{-1} \cdots x_{k}^{-1}$ 
then $y_{1} \cdots y_{n} y_{1}^{-1} \cdots y_{n}^{-1}\left[\left(\psi_{1}, \beta_{1}\right), \ldots,\left(\psi_{k}, \beta_{k}\right)\right]=\left(\psi^{\prime}, \beta^{\prime}\right)$ can be expressed as $\left(\varphi_{1}, \gamma_{1}\right)\left(\varphi_{2}, \gamma_{2}\right)$ where $(\psi, \beta)=\left(\varphi_{2}, \gamma_{2}\right)\left(\varphi_{1}, \gamma_{1}\right)$. If $\{v\}$ $=\mathbf{d} \beta$ then $v \gamma_{2} \in \mathbf{d} \beta^{\prime}$ and $v \gamma_{2} \beta^{\prime}=v \gamma_{2}$ because $v \gamma_{2} \gamma_{1} \gamma_{2}=v \gamma_{2}$ since $v \gamma_{2} \gamma_{1}=v \beta=v$. Then

$$
v \gamma_{2} \psi^{\prime}=\left(v \gamma_{2} \varphi_{1}\right)\left(v \gamma_{2} \gamma_{1} \varphi_{2}\right)=\left(v \gamma_{2} \varphi_{1}\right)\left(v \varphi_{2}\right)=\left(v \varphi_{2}\right)\left(v \gamma_{2} \varphi_{1}\right)
$$

since $C_{m}$ is abelian. But $\left(v \varphi_{2}\right)\left(v \gamma_{2} \varphi_{1}\right)=v \psi$ which is not an idempotent and so, as a result, $\left(\psi^{\prime}, \beta^{\prime}\right)$ is not an idempotent.

(iv) For some $i \in\{1, \ldots, k\},\left(\psi_{i}, \beta_{i}\right)=\left(\varphi_{n}, \alpha_{n}\right)$ or $\left(\varphi_{n}, \alpha_{n}\right)^{-1}$. By (ii), if $\mathbf{d} \beta=\{v\}$ then $v \beta=v$. Therefore, if $(\psi, \beta)$ is not an idempotent then $v \psi$ is not the identity of $C_{m}$. The only elements of $T_{m, n}$ which can contribute non-identity elements to $v \psi$ are those $(\psi, \beta)$ for which $|\mathbf{d} \beta|=1,\left(\phi_{n}, \alpha_{n}\right)$ and $\left(\phi_{n}^{-1}, \alpha_{n}^{-1}\right)$. Now

$$
\begin{aligned}
v \psi= & \left(v \psi_{1}\right)\left(v \beta_{1} \psi_{2}\right) \cdots\left(v \beta_{1} \cdots \beta_{k-1} \psi_{k}\right)\left(v \beta_{1} \cdots \beta_{k} \psi_{1}^{-1}\right) \\
& \left(v \beta_{1} \cdots \beta_{k} \beta_{1}^{-1} \psi_{2}^{-1}\right) \cdots\left(v \beta_{1} \cdots \beta_{k} \beta_{1}^{-1} \cdots \beta_{k-1}^{-1} \psi_{k}^{-1}\right) .
\end{aligned}
$$

If $\left(\psi_{i}, \beta_{i}\right)$ is such that $\left|\mathbf{d} \beta_{i}\right|=1$, then in this factorization of $v \psi, \psi_{i}$ contributes $v \beta_{1} \cdots \beta_{i-1} \psi_{i}=g$, say, and $v \beta_{1} \cdots \beta_{k} \beta_{1}^{-1} \cdots \beta_{i-1}^{-1} \psi_{i}^{-1}=$ $g^{-1}$, since $g^{-1}$ is the only element of $\mathbf{r} \psi_{i}^{-1}$. Thus, the contributions to this factorization of $v \psi$ by $\psi_{i}$ cancel and so, if $(\psi, \beta)$ is not an idempotent, one of the $\left(\psi_{i}, \beta_{i}\right)$ must be $\left(\phi_{n}, \alpha_{n}\right)$ or $\left(\phi_{n}, \alpha_{n}\right)^{-1}$.

(v) None of the $\left(\psi_{i}, \beta_{i}\right)$ is an idempotent. This follows from the general observation that if $e=e^{2}$ and aebec is not an idempotent then $a e b e c=a e a^{-1}(a b c) c^{-1} e c$ and so $a b c$ cannot be an idempotent. Thus, $\left(\psi_{i}, \beta_{i}\right)$ an idempotent contradicts the minimality of $k$.

As a consequence of the aforementioned observations, the following assumptions concerning the $\left(\psi_{i}, \beta_{i}\right)$ can be made. First of all, by (iii) and (iv) we may assume that $\left(\psi_{1}, \beta_{1}\right)=\left(\phi_{n}, \alpha_{n}\right)$. Secondly, assume that the $k$-tuple $\left\langle\left(\psi_{1}, \beta_{1}\right), \ldots,\left(\psi_{k}, \beta_{k}\right)\right\rangle$ contains a maximal number of elements from the kernel of $T_{m, n}$ among the collection of $k$-tuples from $T_{m, n}$ whose first element is $\left(\phi_{n}, \alpha_{n}\right)$ and which witness that $T_{m, n}$ does not satisfy $\tau_{k}$.

There are two stages to the remainder of the proof. The first stage is showing that exactly one of the $\left(\psi_{i}, \beta_{i}\right)$ is a member of the kernel of $T_{m, n}$. We do this in four parts.

(1) For any $i \in\{1, \ldots, k\}$, both $\left(\psi_{i}, \beta_{i}\right)$ and $\left(\psi_{i+1}, \beta_{i+1}\right)$ do not belong to the kernel of $T_{m, n}$.

Suppose that both $\left(\psi_{i}, \beta_{i}\right)$ and $\left(\psi_{i+1}, \beta_{i+1}\right)$ belong to the kernel of $T_{m, n}$. If $\mathbf{d} \beta_{i}=\left\{v_{i}\right\}$ and $\mathbf{d} \beta_{i+1}=\left\{v_{i+1}\right\}$ then $v_{i} \beta_{i}=v_{i+1}$ since 
$\beta_{i} \beta_{i+1} \neq 0$ and $v_{i+1} \beta_{i+1}=v_{i}$ since $\beta_{i}^{-1} \beta_{i+1}^{-1} \neq 0$. It follows that

$$
v_{i} \beta_{i} \beta_{i+1}=v_{i} \text { and } v_{i+1} \beta_{i+1} \beta_{i}=v_{i+1}
$$

and

$$
\begin{aligned}
& \left(v_{i+1} \psi_{i}^{-1}\right)\left(v_{i+1} \beta_{i}^{-1} \psi_{i+1}^{-1}\right)=\left(v_{i} \beta_{i} \psi_{i}^{-1}\right)\left(v_{i} \psi_{i+1}^{-1}\right) \\
& \quad=\left(v_{i} \psi_{i}\right)^{-1}\left(v_{i} \beta_{i+1}^{-1} \psi_{i+1}\right)^{-1} \\
& \quad=\left(v_{i} \psi_{i}\right)^{-1}\left(v_{i+1} \psi_{i+1}\right)^{-1} \\
& \quad=\left(v_{i+1} \psi_{i+1}\right)^{-1}\left(v_{i} \psi_{i}\right)^{-1} \quad \text { (since } C_{m} \text { is abelian) } \\
& \quad=\left[\left(v_{i} \psi_{i}\right)\left(v_{i+1} \psi_{i+1}\right)\right]^{-1} .
\end{aligned}
$$

As a consequence of this we have that

$$
\begin{aligned}
& x_{1} \cdots x_{i-1} x_{i+2} \cdots x_{k} x_{1}^{-1} \cdots x_{i-1}^{-1} x_{i+2}^{-1} \cdots x_{k}^{-1} \\
& \quad\left[\left(\psi_{1}, \beta_{1}\right), \ldots,\left(\psi_{i-1}, \beta_{i-1}\right),\left(\psi_{i+2}, \beta_{i+2}\right), \ldots,\left(\psi_{k}, \beta_{k}\right)\right]
\end{aligned}
$$

is equal to $(\psi, \beta)$, which is not an idempotent by assumption. Thus, $T_{m, n}$ does not satisfy the equation $\tau_{k-2}$, contrary to our choice of $k$. Note that under these conditions, $k \geq 3$, by observation (iv). In the case $k=3$, the conclusion is that $T_{m, n}$ does not satisfy $\tau_{1}$ which is absurd since all inverse semigroups satisfy the equation $x x^{-1} \in E$.

(2) If $\left(\psi_{i}, \beta_{i}\right)$ is an element of the kernel then

(i) if $\mathbf{d} \beta_{i}=\left\{w x_{1} \cdots x_{j} \rho\right\}$, then $w x_{1} \cdots x_{j} \rho \beta_{i}=w x_{1} \cdots x_{n} x_{1}^{-1} \cdots$ $x_{j}^{-1} \rho$

(ii) if $\mathbf{d} \beta_{i}=\left\{w x_{1} \cdots x_{n} x_{1}^{-1} \cdots x_{j}^{-1} \rho\right\}$, then $w x_{1} \cdots x_{n} x_{1}^{-1} \cdots$ $x_{j}^{-1} \rho \beta_{i}=w x_{1} \cdots x_{j} \rho$.

(i) We have assumed that $\left(\psi_{1}, \beta_{1}\right)=\left(\phi_{n}, \beta_{n}\right)$ and so $i \neq 1$. Let $\mathbf{d} \beta_{i-1}=\left\{v_{1}, v_{2}\right\}$ (by (1) $\left.\left|\mathbf{d} \beta_{i-1}\right|=2\right)$, and suppose that $v_{1} \beta_{i-1}=u_{1}$ and $v_{2} \beta_{i-1}=u_{2}$. Now, $\beta_{i-1} \beta_{i} \neq 0$ so one of $u_{1}$ and $u_{2}$ must be $w x_{1} \cdots x_{j} \rho$, say $u_{1}=w x_{1} \cdots x_{j} \rho$. Also, $\beta_{i-1}^{-1} \beta_{i}^{-1} \neq 0$ so one of $v_{1}$ and $v_{2}$ must be $w x_{1} \cdots x_{j} \rho \beta_{i}$. If $v_{1}=w x_{1} \cdots x_{j} \rho \beta_{i}$ then $\left(\psi_{i-1}, \beta_{i-1}\right)$ can be replaced by $(\hat{\psi}, \hat{\beta})$ where $\mathbf{d} \hat{\beta}=\left\{v_{1}\right\}$ and $v_{1} \hat{\beta}=$ $u_{1}$ and $v_{1} \hat{\psi}=v_{1} \psi_{i-1}$. This new substitution witnesses that $T_{m, n}$ does not satisfy $\tau_{k}$. Following the argument in (1) above, we obtain that $T_{m, n}$ does not satisfy $\tau_{k-2}$, contradicting the minimality of $k_{3}$. Thus, $v_{2}=w x_{1} \cdots x_{j} \rho \beta_{i}$. By observation (v), $\beta_{i-1}$ is $\alpha_{p}$ or $\alpha_{p}^{-1}$ for some $p \in\{1, \ldots, n\}$.

If $\beta_{i-1}=\alpha_{p}$ then $v_{1} \beta_{i-1}=w x_{1} \cdots x_{j} \rho$ implies that $v_{1} x_{p} \rho=$ $w x_{1} \cdots x_{j} \rho$ and hence that either $p=j$ and $v_{1} \rho w x_{1} \cdots x_{j-1}$ or $j=n, p=1$ and $v_{1} \rho w x_{1} \cdots x_{n} x_{1}^{-1}$. Thus, $w x_{1} \cdots x_{j} \rho \beta_{i}=v_{2}=$ 
$w x_{1} \cdots x_{n} x_{1}^{-1} \cdots x_{j}^{-1} \rho$, by the definition of $\alpha_{p}$ or $w x_{1} \cdots x_{n} \rho \beta_{i}=$ $v_{2}=w \rho$, which is what we want to prove.

If $\beta_{i-1}=\alpha_{p}^{-1}$ then $v_{1} \beta_{i-1}=w x_{1} \cdots x_{j} \rho$ implies that $v_{1} x_{p}^{-1} \rho=$ $w x_{1} \cdots x_{j} \rho$ and hence that $v_{1} \rho w x_{1} \cdots x_{p}$ and $p=j+1$. Note that in this case $j \neq n$ since if $u$ is an initial segment of $w$, then $w u x_{p}^{-1} \rho w x_{1} \cdots x_{n}$ is impossible by Lemma 3.5. Therefore, $w x_{1} \cdots x_{j} \rho \beta_{i}=v_{2}=w x_{1} \cdots x_{n} x_{1}^{-1} \cdots x_{p-1}^{-1} \rho w x_{1} \cdots x_{n} x_{1}^{-1} \cdots x_{j}^{-1}$, by the definition of $\alpha_{p}^{-1}$.

(ii) As in (i) we can assume that $\mathbf{d} \beta_{i-1}=\left\{v_{1}, w x_{1} \cdots x_{n} x_{1}^{-1} \ldots\right.$ $\left.x_{j}^{-1} \rho \beta_{i}\right\}$ and that $v_{1} \beta_{i-1}=w x_{1} \cdots x_{n} x_{1}^{-1} \cdots x_{j}^{-1} \rho$. Again, by observation (v), we may assume that $\beta_{i-1}=\alpha_{p}$ or $\alpha_{p}^{-1}$.

If $\beta_{i-1}=\alpha_{p}$ then $v_{1} x_{p} \rho=w x_{1} \cdots x_{n} x_{1}^{-1} \cdots x_{j}^{-1} \rho$ and hence $p=$ $j+1$ and $v_{1} \rho w x_{1} \cdots x_{n} x_{1}^{-1} \cdots x_{j+1}^{-1}$. Note that if $j=n, w x_{1} \cdots x_{n} x_{1}^{-1}$ $\cdots x_{j}^{-1} \rho w$ and so for any initial segment $u$ of $w, w u x_{p} \rho w$ is impossible, by Lemma 3.5. Therefore, by the definition of $\alpha_{p}$, $w x_{1} \cdots x_{n} x_{1}^{-1} \cdots x_{j}^{-1} \rho \beta_{i}=w x_{1} \cdots x_{j} \rho$.

If $\beta_{i-1}=\alpha_{p}^{-1}$ then $v_{1} x_{p}^{-1} \rho=w x_{1} \cdots x_{n} x_{1}^{-1} \cdots x_{j}^{-1} \rho$ and so $p=j$ and $v_{1} \rho w x_{1} \cdots x_{n} x_{1}^{-1} \cdots x_{n} x_{1}^{-1} \cdots x_{j-1}^{-1}$ or $j=n, p=1, v_{1} \rho w x_{1}$. By the definition of $\alpha_{p}^{-1}, w x_{1} \cdots x_{n} x_{1}^{-1} \cdots x_{j}^{-1} \rho \beta_{i}=w x_{1} \cdots x_{j} \rho$ and if $j=n, p=1, w \rho \beta_{i}=v_{2}=w x_{1} \cdots x_{n} \rho$.

(3) At most one of the $\left(\psi_{i}, \beta_{i}\right)$ belongs to the kernel of $T_{m, n}$.

Suppose that $\left(\psi_{j}, \beta_{j}\right)$ and $\left(\psi_{j+p}, \beta_{j+p}\right)$ are two members of the kernel of $T_{m, n}$ and they are the first two such elements appearing in the sequence $\left\{\left(\psi_{1}, \beta_{1}\right), \ldots,\left(\psi_{k}, \beta_{k}\right)\right\}$. Let $\mathbf{d} \beta_{j}=\left\{v_{1}\right\}, \mathbf{d} \beta_{j+p}=$ $\left\{u_{1}\right\}, v_{1} \beta_{j}=v_{2}$ and $v_{1} \psi_{j}=g_{1}$, and $u_{1} \beta_{j+p}=u_{2}$ and $u_{1} \psi_{j+p}=g_{2}$. The claim is that if $(\psi, \beta)$ is not an idempotent then neither is the following:

$$
\begin{gathered}
x_{1} \cdots x_{j-1} x_{j+1}^{-1} \cdots x_{j+p-1}^{-1} x_{j+p+1} \cdots x_{k} x_{1}^{-1} \\
\quad \cdots x_{j-1}^{-1} x_{j+1} \cdots x_{j+p-1} x_{j+p+1}^{-1} \cdots x_{k}^{-1}
\end{gathered}
$$

when $\left(\psi_{i}, \beta_{i}\right)$ is substituted for $x_{i}$ for all $x_{i}$ appearing in the expression. Call this element $\left(\psi^{\prime}, \beta^{\prime}\right)$. If the claim is correct then $T_{m, n}$ does not satisfy $\tau_{k-2}$, contrary to our assumptions. We first show that $\mathbf{d} \beta^{\prime} \supseteq \mathbf{d} \beta$ and $\beta^{\prime}$ equals $\beta$ on $\mathbf{d} \beta$. Now, with $\mathbf{d} \beta=\{v\}$,

$$
\begin{aligned}
& v \beta_{1} \cdots \beta_{j-1}=v_{1} ; \\
& v_{1} \in \mathbf{d} x_{j+1}^{-1} \cdots x_{j+p-1}^{-1}\left[\left(\psi_{j+1}, \beta_{j+1}\right), \ldots,\left(\psi_{j+p-1}, \beta_{j+p-1}\right)\right] \text { and } \\
& v_{1} \beta_{j+1}^{-1} \cdots \beta_{j+p-1}^{-1}=u_{2} ;
\end{aligned}
$$




$$
\begin{aligned}
& u_{2} \in \mathbf{d} x_{j+p+1} \cdots x_{k} x_{1}^{-1} \cdots x_{j-1}^{-1}\left[\left(\psi_{j+p+1}, \beta_{j+p+1}\right), \ldots,\left(\psi_{k}, \beta_{k}\right),\right. \\
& \left.\quad\left(\psi_{1}, \beta_{1}\right), \ldots,\left(\psi_{j-1}, \beta_{j-1}\right)\right] \\
& u_{2} \beta_{j+p+1} \cdots \beta_{k} \beta_{1}^{-1} \cdots \beta_{j-1}^{-1}=v_{2} ; \\
& v_{2} \in \mathbf{d} x_{j+1} \cdots x_{j+p-1}\left[\left(\psi_{j+1}, \beta_{j+1}\right), \ldots,\left(\psi_{j+p-1}, \beta_{j+p-1}\right)\right] \quad \text { and } \\
& v_{2} \beta_{j+1} \cdots \beta_{j+p-1}=u_{1} ; \\
& u_{1} \in \mathbf{d} x_{j+p+1}^{-1} \cdots x_{k}^{-1}\left[\left(\psi_{j+p+1}, \beta_{j+p+1}\right), \ldots,\left(\psi_{k}, \beta_{k}\right)\right] \quad \text { and } \\
& u_{1} \beta_{j+p+1}^{-1} \cdots \beta_{k}^{-1}=v \beta=v .
\end{aligned}
$$

Thus, $v \in \mathbf{d} \beta^{\prime}$ and $v \beta^{\prime}=v \beta=v$. By calculation one sees that $v \psi$ must be equal to $v \psi^{\prime} g_{1} g_{2} g_{1}^{-1} g_{2}^{-1}$, since $C_{m}$ is abelian, and thus, $v \psi=v \psi^{\prime}$. Therefore, if $(\psi, \beta)$ is not an idempotent, then neither is $\left(\psi^{\prime}, \beta^{\prime}\right)$. It now follows that at most one of the $\left(\psi_{i}, \beta_{i}\right)$ belongs to the kernel of $T_{m, n}$.

(4) Exactly one of the $\left(\psi_{i}, \beta_{i}\right)$ is a member of the kernel of $T_{m, n}$.

First of all, observe that if none of the $\left(\psi_{i}, \beta_{i}\right)$ belongs to the kernel then each $\left(\psi_{i}, \beta_{i}\right)$ is $\left(\phi_{p}, \alpha_{p}\right)$ or $\left(\phi_{p}, \alpha_{p}\right)^{-1}$ for some $p$. By the definition of the $\alpha_{p}$, if $v \beta_{1} \cdots \beta_{k} \in \mathbf{d} \beta_{1}^{-1}$ then $v \beta_{1} \cdots \beta_{k} \beta_{1}^{-1}=$ $v$. This is because if $v=w u \rho$ for some initial segment $u$ of $w$ then $v \beta_{1} \cdots \beta_{k}=w u^{\prime} \rho$ for some initial segment $u^{\prime}$ of $w$ and the difference between the lengths of $u$ and $u^{\prime}$ is not greater than $k$ and hence strictly less than $n$. It follows that $v \beta_{1} \cdots \beta_{k}$ must be $v \beta_{1}$. By the same reasoning we can conclude that, for all $1 \leq i \leq$ $k, v \beta_{1} \cdots \beta_{k} \beta_{1}^{-1} \cdots \beta_{i}^{-1}=v \beta_{1} \cdots \beta_{i-1}$. Since $\mathbf{d} \beta=\{v\}$, we can replace each $\left(\psi_{i}, \beta_{i}\right)$ with an element of the kernel and conclude that if $(\psi, \beta)$ is not an idempotent then neither is the result of this new substitution. But this cannot be since the kernel of $T_{m, n}$ is a Brandt semigroup over an abelian group and so satisfies the equation $\tau_{k}$. Therefore, exactly one of the $\left(\psi_{i}, \beta_{i}\right)$ belongs to the kernel of $T_{m, n}$. This completes the first stage of the proof.

Let $\left(\psi_{j}, \beta_{j}\right)$ be the only member of $\left\{\left(\psi_{1}, \beta_{1}\right), \ldots,\left(\psi_{k}, \beta_{k}\right)\right\}$ which belongs to the kernel of $T_{m, n}$. Let $\mathbf{d} \beta_{j}=\left\{v_{1}\right\}, v_{1} \beta_{j}=v_{2}$ and $v_{1} \psi_{j}=g_{1}$. We consider the following two cases: (i) $v_{1} \rho w x_{1} \cdots x_{p}$; and (ii) $v_{1} \rho w x_{1} \cdots x_{n} x_{1}^{-1} \cdots x_{p}^{-1}$.

(i) If $v_{1} \rho w x_{1} \cdots x_{p}$ then $v_{2}=w x_{1} \cdots x_{n} x_{1}^{-1} \cdots x_{p}^{-1} \rho$ by the first stage, part (2). Since $\left(\psi_{1}, \beta_{1}\right)=\left(\phi_{n}, \alpha_{n}\right)$ and $k<n$, by the constraints on the $\left(\psi_{i}, \beta_{i}\right)$ discussed thus far, for some $1<q<j$, $\left(\psi_{q}, \beta_{q}\right)=\left(\phi_{n}, \alpha_{n}\right)^{-1}$. [That is, because for $i=1, \ldots, j-1$, 
$\left(\psi_{i}, \beta_{i}\right)$ is either $\left(\phi_{h}, \alpha_{h}\right)$ or $\left(\phi_{h}, \alpha_{h}\right)^{-1}$, for some $h$, and the projection map of $T_{m, n}$ onto its second coordinate has image $S\left(\tau_{n}\right)$, we have that $v \beta_{1} \beta_{2} \cdots \beta_{j-1}=v x_{i_{1}} x_{i_{2}} \cdots x_{i_{j-1}} \rho$, for some $x_{i_{1}}, x_{i_{2}}, \ldots$, $x_{i_{j-1}} \in X \cup X^{-1}$, and that $x_{i_{1}} x_{i_{2}} \cdots x_{i_{j-1}}$ labels a path in the Schützenberger graph of $x_{1} \cdots x_{n} x_{1}^{-1} \cdots x_{n}^{-1} \rho$ from $v$ to $w x_{1} \cdots x_{p} \rho$. Since $j-1<k<n$, this path must traverse the edge labelled $x_{n}^{-1}$ with terminal vertex $v$. Thus, for some $1<q<j,\left(\psi_{q}, \beta_{q}\right)=$ $\left(\phi_{n}, \alpha_{n}\right)^{-1}$.] Assume that $q$ is the least such integer. Because $k<n$ and each of the $\left(\psi_{i}, \beta_{i}\right)$ is either $\left(\phi_{h}, \alpha_{h}\right)$ or $\left(\phi_{h}, \alpha_{h}\right)^{-1}$, for some $h$, for $1<i \leq q$, as a consequence of the definitions of the $\left(\phi_{h}, \alpha_{h}\right)$, we have that $v \beta_{1} \cdots \beta_{q}=v$ and $\left(v \psi_{1}\right)\left(v \beta_{1} \psi_{2}\right) \cdots$ $\left(v \beta_{1} \cdots \beta_{q-1} \psi_{q}\right)=1$. In a likewise manner we obtain that

$$
\left(v \beta_{1} \cdots \beta_{k}\right) \beta_{1}^{-1} \cdots \beta_{q}^{-1}=v \beta_{1} \cdots \beta_{k}
$$

and

$$
\begin{aligned}
& {\left[\left(v \beta_{1} \cdots \beta_{k}\right) \psi_{1}^{-1}\right]\left[\left(v \beta_{1} \cdots \beta_{k}\right) \beta_{1}^{-1} \psi_{2}^{-1}\right]} \\
& \cdots\left[\left(v \beta_{1} \cdots \beta_{k}\right) \beta_{1}^{-1} \cdots \beta_{q-1}^{-1} \psi_{q}^{-1}\right]=1
\end{aligned}
$$

As a result, $x_{q+1} \cdots x_{k} x_{q+1}^{-1} \cdots x_{k}^{-1}\left[\left(\psi_{q+1}, \beta_{q+1}\right), \ldots,\left(\psi_{k}, \beta_{k}\right)\right]$ is not an idempotent if $(\psi, \beta)$ is not an idempotent, contrary to our choice of $k$.

(ii) If $v_{1} \rho w x_{1} \cdots x_{n} x_{1}^{-1} \cdots x_{p}^{-1}$ then $v_{2} \rho w x_{1} \cdots x_{p}$. Using a similar argument to that used in (i) above, we can assume that $\left(\psi_{1}, \beta_{1}\right)$ is the only $\left(\psi_{i}, \beta_{i}\right)$ equal to $\left(\phi_{n}, \alpha_{n}\right)$ for $i<j$. Moreover, the same argument can be used to show that at most one of the $\left(\psi_{i}, \beta_{i}\right)$ is equal to $\left(\phi_{n}, \alpha_{n}\right)$ for $j<i \leq k$. In this case, by the constraints on the $\left(\psi_{i}, \beta_{i}\right)$ and the definitions of the $\left(\phi_{i}, \alpha_{i}\right)$ and their inverses, $\left(\psi_{k}, \beta_{k}\right)$ is equal to $\left(\phi_{n}, \alpha_{n}\right)$. Thus, the only $\left(\psi_{i}, \beta_{i}\right)$ equal to $\left(\phi_{n}, \alpha_{n}\right)$ are $\left(\psi_{1}, \beta_{1}\right)$ and $\left(\psi_{k}, \beta_{k}\right)$. But for any inverse semigroup, $a x a a^{-1} y a^{-1}$ is not an idempotent implies that $x y$ is not an idempotent. It would then follow that $T_{m, n}$ does not satisfy the equation $\tau_{k-2}$, a contradiction.

Since every inverse semigroup satisfies $\tau_{1}$, the proof is complete if we can show that, for $n>2, T_{m, n}$ satisfies $\tau_{2}$. This is not difficult to verify directly: Suppose that $(\psi, \beta) \in T_{m, n}$ is such that $\left(\phi_{n}, \alpha_{n}\right)(\psi, \beta)\left(\phi_{n}, \alpha_{n}\right)^{-1}(\psi, \beta)^{-1}$ is not an idempotent. Since $\mathscr{B}^{1}$ does satisfy $\tau_{2}$, we have that $\alpha_{n} \beta \alpha_{n}^{-1} \beta^{-1}$ is an idempotent. Thus, for all $v \in \mathbf{d} \alpha_{n} \beta \alpha_{n}^{-1} \beta^{-1} \subseteq \mathbf{d} \alpha_{n}, v \alpha_{n} \beta \alpha_{n}^{-1} \beta^{-1}=v$. Therefore, both 
$v$ and $v \alpha_{n}$ (which are not equal) are in the domain of $\beta$. For either $v$ in the domain of $\alpha_{n}$, there is no pair $(\psi, \beta)$ in $T_{m, n}$ such that $\mathbf{d} \beta=\left\{v, v \alpha_{n}\right\}$. It follows that $T_{m, n}$ must satisfy $\tau_{2}$.

LEMMA 4.5. Let $m, n \in \omega, m, n \geq 2 . T_{m, n}^{1}$ satisfies the equation $\tau_{k}$ for $k<n$, but $T_{m, n}^{1}$ does not satisfy the equation $\tau_{k}$ for $k \geq n$.

Proof. This is an immediate consequence of Lemmas 4.4 and 4.3.

REMARK. The only property of the varieties $\mathscr{A}_{m}$ that we used in the construction of the $T_{m}$, 's was that they each satisfied the equations $\tau_{n}, n \in \omega$. This is also true of the variety groups. Thus, in a similar way, we can construct a family of inverse semigroups $\left\{T_{n}^{1}\right\}$ such that, for each $n, T_{n}^{1}$ satisfies the equations $\tau_{k}$, for $k<n$, but $T_{n}^{1}$ does not satisfy the equations $\tau_{k}$, for $k \geq n$. Moreover, for each $n \in \omega, \mathscr{A} \vee \mathscr{B}^{1} \subseteq\left\langle T_{n}^{1}\right\rangle \subseteq \mathscr{A} \circ \mathscr{B}^{1}$.

5. A class of varieties in the interval $\left[\mathscr{A}_{m}, \mathscr{B}^{1}\right]$. The inverse semigroups defined in the previous section can be used to define an infinite collection of varieties in the interval $\left[\mathscr{A}_{m}, \mathscr{B}^{1}\right]$. Once it is established that the interval $\left[\mathscr{A}_{m}, \mathscr{B}^{1}\right]$ is infinite, it can then be shown that other intervals which coincide with $\nu$-classes are infinite.

Notation 5.1. Let $m \in \omega$. For each $n \in \omega$, define the variety $\mathscr{V}_{m, n}$ to be the variety of inverse semigroups generated by $\left\{T_{m, k}^{1}: k \geq\right.$ $n\}$.

Proposition 5.2. Let $m, n \in \omega$, with $m, n>1$.

(a) $\mathscr{V}_{m, n}$ satisfies $\tau_{j}$ for $j<n$;

(b) $\mathscr{V}_{m, n}$ does not satisfy $\tau_{j}$ for $j \geq n$;

(c) $\mathscr{V}_{m, n} \supset \mathscr{V}_{m, n+1}$ (the containment is proper).

Proof. (a) By Lemma 4.5, $T_{m, k}^{1}$ satisfies $\tau_{j}$ for $j<k$. Therefore, each generator of $\mathscr{V}_{m, n}$ satisfies $\tau_{j}$ for $j<n$, and hence $\mathscr{V}_{m, n}$ satisfies $\tau_{j}$ for $j<n$.

(b) By Lemma 4.3, $T_{m, j}^{1}$ does not satisfy $\tau_{j}$. Since $T_{m, j}^{1}, j \geq n$, is a generator of $\mathscr{V}_{m, n}$, the equation $\tau_{j}$ is not satisfied by $\mathscr{V}_{m, n}$, for all $j \geq n$.

(c) $\left\{T_{m, k}^{1}: k \geq n\right\} \supset\left\{T_{m, k}^{1}: k \geq n+1\right\}$ and so $\mathscr{V}_{m, n}=\left\langle T_{m, k}^{1}: k \geq\right.$ $n\rangle \supset\left\langle T_{m, k}^{1}: k \geq n+1\right\rangle=\mathscr{V}_{m, n+1}$. That the containment is proper follows from parts (a) and (b). 
As a consequence of Proposition 5.2, the collection of varieties of inverse semigroups $\left\{\mathscr{V}_{m, n}: n>1\right\}$ forms an infinite chain in the lattice of varieties of inverse semigroups. Furthermore, by Lemma 4.2, $\mathscr{A}_{m} \vee \mathscr{B}^{1} \subseteq \mathscr{V}_{m, n} \subseteq \operatorname{Wr}\left(\mathscr{A}_{m}, \mathscr{B}^{1}\right)$. Since $\operatorname{Wr}\left(\mathscr{A}_{m}, \mathscr{B}^{1}\right)=\mathscr{A}_{m} \circ \mathscr{B}^{1}$, and the $\nu$-class of $\mathscr{A}_{m} \vee \mathscr{B}^{1}$ is the interval $\left[\mathscr{A}_{m} \vee \mathscr{B}^{1}, \mathscr{A}_{m} \circ \mathscr{B}^{1}\right.$ ], we have the following result.

THEOREM 5.3. The $\nu$-class of the variety $\mathscr{A}_{m} \vee \mathscr{B}^{1}$ possesses an infinite descending chain of varieties.

Using Theorem 5.3, we can show that other intervals in $\mathscr{L}(\mathscr{I})$ are infinite.

Lemma 5.4. Let $\mathscr{V} \in\left[\mathscr{A}_{m} \vee \mathscr{B}^{1}, \mathscr{A}_{n} \circ \mathscr{B}^{1}\right]$, where $\mathscr{A}_{m}$ is the variety of abelian groups of exponent $m$, and let $\mathscr{U} \in\left[\mathscr{A}_{m} \vee \mathscr{B}^{1}, \mathscr{A}_{m}^{\max }\right]$. Then$$
\operatorname{ker} \rho(\mathscr{U} \vee \mathscr{V})=\operatorname{ker} \rho(\mathscr{V}) \text { and } \operatorname{tr} \rho(\mathscr{U} \vee \mathscr{V})=\operatorname{tr} \rho(\mathscr{U}) \text {. }
$$

Proof. $\mathscr{A}_{m} \subseteq \mathscr{V}$ and so $\mathscr{A}_{m}^{\max } \subseteq \mathscr{V}^{\max }$. Therefore,

$$
\mathscr{V} \subseteq \mathscr{U} \vee \mathscr{V} \subseteq \mathscr{A}_{m}^{\max } \vee \mathscr{V} \subseteq \mathscr{V}^{\max } \vee \mathscr{V}=\mathscr{V}^{\max }
$$

Since $\operatorname{ker} \rho(\mathscr{V})=\operatorname{ker} \rho\left(\mathscr{V}^{\max }\right)$, it follows that $\operatorname{ker} \rho(\mathscr{U} \vee \mathscr{V})=$ $\operatorname{ker} \rho(\mathscr{V})$.

Also,

$$
\mathscr{U} \subseteq \mathscr{U} \vee \mathscr{V} \subseteq \mathscr{U} \vee \mathscr{V} \vee \mathscr{G}=\mathscr{U} \vee\left(\mathscr{A}_{m} \vee \mathscr{B}^{1}\right) \vee \mathscr{G}=\mathscr{U} \vee \mathscr{G}
$$

Since $\operatorname{tr} \rho(\mathscr{U})=\operatorname{tr} \rho(\mathscr{U} \vee \mathscr{G})$, we have that $\operatorname{tr} \rho(\mathscr{U} \vee \mathscr{V})=\operatorname{tr} \rho(\mathscr{U})$.

Theorem 5.5. Let $\mathscr{U} \in\left[\mathscr{A}_{m} \vee \mathscr{B}^{1}, \mathscr{A}_{m}^{\max }\right]$. Then the interval $\left[\mathscr{U},\left(\mathscr{A}_{m} \circ \mathscr{B}^{1}\right) \vee \mathscr{U}\right]$ contains an infinite descending chain.

Proof. The function $\theta:\left[\mathscr{A}_{m} \vee \mathscr{B}^{1}, \mathscr{A}_{m} \circ \mathscr{B}^{1}\right] \rightarrow\left[\mathscr{U},\left(\mathscr{A}_{m} \circ \mathscr{B}^{1}\right) \vee \mathscr{U}\right]$ defined by $\mathscr{V} \theta=\mathscr{V} \vee \mathscr{U}$ is one-to-one on $\left[\mathscr{A}_{m} \vee \mathscr{B}^{1}, \mathscr{A}_{m} \circ \mathscr{B}^{1}\right]$ by Lemma 5.4 and the fact that all varieties $\mathscr{V}$ in this interval are such that $\operatorname{tr} \rho(\mathscr{V})=\operatorname{tr} \rho\left(\mathscr{A}_{m} \vee \mathscr{B}^{1}\right)$. Clearly $\theta$ is order-preserving, and the result follows from Theorem 5.3.

COROLlary 5.6. Let $\mathscr{U}$ be a combinatorial variety contained in $\mathscr{A}_{m}^{\max }$ and containing $\mathscr{B}^{1}$. Then the $\nu$-class of $\mathscr{U} \vee \mathscr{A}_{m}$, that is, $\left[\mathscr{U} \vee \mathscr{A}_{m}, \mathscr{A}_{m} \circ \mathscr{U}\right]$, contains an infinite descending chain.

Proof. By Theorem 5.5, since $\mathscr{U} \vee \mathscr{A}_{m} \in\left[\mathscr{A}_{m} \vee \mathscr{B}^{1}, \mathscr{A}_{m}^{\text {max }}\right]$ and $\left(\mathscr{A}_{m} \circ \mathscr{B}^{1}\right) \vee \mathscr{U} \subseteq \mathscr{A}_{m} \circ \mathscr{U}$. 
REMARK. The results of this section are true for the variety $\mathscr{S}$ as well. That is, if $\mathscr{V}_{n}$ denotes the variety of inverse semigroups generated by $\left\{T_{n}^{1}: k \geq n\right\}$, the analogous results to Proposition 5.2 hold and the remaining results of this section are true when we replace $\mathscr{A}_{m}$ by $\mathscr{A}$.

\section{REFERENCES}

[B] J. L. Bales, On product varieties of inverse semigroups, J. Austral. Math. Soc., 28 (1979), 107-119.

[BS] S. Burris and H. P. Sankappanavar, A Course in Universal Algebra, GTM 78, Springer-Verlag, New York (1981).

[C] D. F. Cowan, A class of varieties of inverse semigroups, to appear in J. Algebra.

[H] C. H. Houghton, Embedding inverse semigroups in wreath products, Glasgow Math. J., 17 (1976), 77-82.

[K1] E. I. Kleiman, On the basis of identities of Brandt semigroups, Semigroup Forum, 13 (1977), 209-218.

[K2] _ Bases of identities of varieties of inverse semigroups, Sibirsk. Matem. Zh., 20 (1979), 760-777 (Russian).

[P] M. Petrich, Inverse Semigroups, Wiley, New York (1984).

[PR] M. Petrich and N. R. Reilly, E-unitary covers and varieties of inverse semigroups, Acta Sci. Math., Szeged, 46 (1983), 59-72.

[R1] N. R. Reilly, Modular sublattices of the lattice of varieties of inverse semigroups, Pacific J. Math., 89 (1980), 405-417.

[R2] _ Varieties of completely semisimple inverse semigroups, J. Algebra, 65 (1980), 427-444.

[S] J. B. Stephen, Presentations of inverse monoids, J. Pure Appl. Algebra, 63 (1990), 81-112.

Received February 10, 1990 and in revised form May 11, 1990.

SIMON FRASER UNIVERSITY

BURNABY, BC CANADA V5A 1S6 


\section{PACIFIC JOURNAL OF MATHEMATICS EDITORS}

\author{
V. S. VARADARAJAN \\ (Managing Editor) \\ University of California \\ Los Angeles, CA 90024-1555-05 \\ Herbert Clemens \\ University of Utah \\ Salt Lake City, UT 84112 \\ THOMAS ENRIGHT \\ University of California, San Diego \\ La Jolla, CA 92093
}

Nicholas ERCOLANI

University of Arizona

Tucson, AZ 85721

R. FINN

Stanford University

Stanford, CA 94305

VAUGHAN F. R. JONES

University of California

Berkeley, CA 94720

STEVEN KeRCKHOFF

Stanford University

Stanford, CA 94305
C. C. MOORE

University of California

Berkeley, CA 94720

Martin ScharlemanN

University of California

Santa Barbara, CA 93106

HAROLd STARK

University of California, San Diego

La Jolla, CA 92093

\section{ASSOCIATE EDITORS}
R. ARENS
E. F. BECKENBACH
B. H. NeUMANN
F. WolF
K. Yoshida (1906-1982)
(1904-1989)

SUPPORTING INSTITUTIONS

UNIVERSITY OF ARIZONA

UNIVERSITY OF BRITISH COLUMBIA

CALIFORNIA INSTITUTE OF TECHNOLOGY

UNIVERSITY OF CALIFORNIA

MONTANA STATE UNIVERSITY

UNIVERSITY OF NEVADA, RENO

NEW MEXICO STATE UNIVERSITY

OREGON STATE UNIVERSITY
UNIVERSITY OF OREGON

UNIVERSITY OF SOUTHERN CALIFORNIA

STANFORD UNIVERSITY

UNIVERSITY OF HAWAII

UNIVERSITY OF TOKYO

UNIVERSITY OF UTAH

WASHINGTON STATE UNIVERSITY

UNIVERSITY OF WASHINGTON 


\section{Pacific Journal of Mathematics}

\section{Vol. 151, No. $1 \quad$ November, 1991}

Fernanda Maria Botelho, Rotational entropy for annulus endomorphisms . . . 1

David F. Cowan, Some infinite chains in the lattice of varieties of inverse

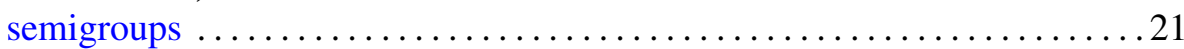

S. Greco and G. Raciti, The Lüroth semigroup of plane algebraic curves . . . 44

Kang-Tae Kim, Automorphism groups of certain domains in $\mathbf{C}^{n}$ with a

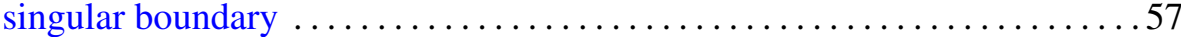

R. James Milgram, Surgery with finite fundamental group. I: The

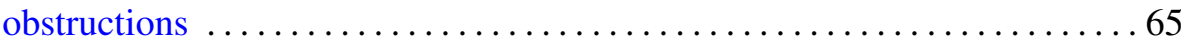

R. James Milgram, Surgery with finite fundamental group. II: The oozing

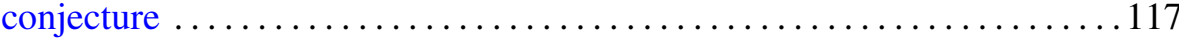

Shahriar Shahriari, On central type factor groups $\ldots \ldots \ldots \ldots \ldots \ldots \ldots 151$

Lynne Walling, Hecke eigenforms and representation numbers of quadratic forms ............................................... 179 